\title{
Effects of Symmetric and Asymmetric Nonlinearity on the Dynamics of a Third-Order Autonomous Duffing-Holmes Oscillator
}

\author{
Isaac Sami Doubla ${ }^{D},{ }^{1,2}$ Jacques Kengne, ${ }^{1}$ Raoul Blaise Wafo Tekam, \\ Zeric Tabekoueng Njitacke $\mathbb{D}^{1,4}{ }^{1,4}$ and Clotaire Thierry Sanjong Dagang ${ }^{1}$ \\ ${ }^{1}$ Unité de Recherche d'Automatique et Informatique Appliquée (URAIA), Department of Electrical Engineering, \\ IUT-FV Bandjoun, University of Dschang, Dschang, Cameroon \\ ${ }^{2}$ Unité de Recherche de Matière Condensée, d'Electronique et de Traitement du Signal (URAMACETS), Department of Physics, \\ University of Dschang, P.O. Box 67, Dschang, Cameroon \\ ${ }^{3}$ Laboratory of Mechanics, Department of Physics, Faculty of Science, University of Yaoundé I, P.O. Box 812, Yaoundé, Cameroon \\ ${ }^{4}$ Department of Electrical and Electronic Engineering, College of Technology (COT), University of Buea, P.O. Box 63, \\ Buea, Cameroon
}

Correspondence should be addressed to Isaac Sami Doubla; samyisac@yahoo.fr

Received 24 September 2020; Revised 27 October 2020; Accepted 2 December 2020; Published 14 December 2020

Academic Editor: Chun-Biao Li

Copyright (c) 2020 Isaac Sami Doubla et al. This is an open access article distributed under the Creative Commons Attribution License, which permits unrestricted use, distribution, and reproduction in any medium, provided the original work is properly cited.

\begin{abstract}
A generalized third-order autonomous Duffing-Holmes system is proposed and deeply investigated. The proposed system is obtained by adding a parametric quadratic term $\left(m x^{2}\right)$ to the cubic nonlinear term $\left(-x^{3}\right)$ of an existing third-order autonomous Duffing-Holmes system. This modification allows the system to feature smoothly adjustable nonlinearity, symmetry, and nontrivial equilibria. A particular attention is given to the effects of symmetric and asymmetric nonlinearity on the dynamics of the system. For the specific case of $m=0$, the system is symmetric and interesting phenomena are observed, namely, coexistence of symmetric bifurcations, presence of parallel branches, and the coexistence of four (periodic-chaotic) and six (periodic) symmetric attractors. For $m \neq 0$, the system loses its symmetry. This favors the emergence of other behaviors, such as the coexistence of asymmetric bifurcations, involving the coexistence of several asymmetric attractors (periodic-periodic or periodicchaotic). All these phenomena have been numerically highlighted using nonlinear dynamic tools (bifurcation diagrams, Lyapunov exponents, phase portraits, time series, frequency spectra, Poincaré section, cross sections of the attraction basins, etc.) and an analog computer of the system. In fact, PSpice simulations of the latter confirm numerical results. Moreover, amplitude control and synchronization strategies are also provided in order to promote the exploitation of the proposed system in engineering.
\end{abstract}

\section{Introduction}

The study of nonlinear dynamic systems has been attracting researchers from various fields of science. Thanks to analytical, numerical, and experimental tools, striking nonlinear phenomena involved for instance in the evolution of stock market [1], neural networks [2-6], and population dynamics [7] have been uncovered. However, systems exhibiting chaotic dynamics are of particular interest owing to their potential applications in various fields of science and engineering such as image encryption [8], random bits generation [9], and secure communications [10]. Yet, transient chaos [11, 12], antimonotonicity [13-15], multistability [13-16], and many other dynamical features of chaotic systems are relevant for their applications $[15,17-21]$.

Multistability, namely, the occurrence of two or more attractors for a given set of system parameter values due to initial states (or/and noise sensitivity) [22-24], has explained a large number of events in biology $[25,26]$ and chemistry 
[27], just to mention a few. Some important works based on the construction and study of multistable chaotic systems or chaotic systems with the coexistence of multiple attractors have recently been published in [28-33]. The work of Lai et al. [28] was based on the study of an extended Lü system; their results showed that the system has a pair of strange attractors, a pair of limit cycles, and a pair of point attractors for different initial conditions. The authors [29] have constructed an extremely simple chaotic system with an infinite number of coexisting chaotic attractors. Lai et al. [30] reported a new chaotic system they generated from the simplest chaotic memristor circuit by introducing a simple nonlinear feedback control input. The main characteristic of the new system is that it has an infinite number of coexisting equilibria and abundant attractors. The results of [31] report a new chaotic system without equilibrium with hidden attractors and coexisting attractors. This special feature (multistability) can sometimes be justified by the fact that the system is symmetric [34]. In this line, Kengne and collaborators have highlighted the coexistence of four, six, and eight attractors from a cubic, hyperbolic tangent, and hyperbolic tangent-cubic nonlinearity-based chaotic jerk system, respectively [35-37]. The authors of [38] showed the presence of nine coexisting attractors in a hyperjerk chaotic system with hyperbolic sine nonlinearity. On the other hand, Negou and Kengne [34] recently introduced a jerk system with adjustable symmetry and nonlinearity. The system was obtained by replacing a hyperbolic sine nonlinearity in an existing jerk system with a smoothly adjustable nonlinearity. This modification demonstrated the relevance of adjustable symmetries in the emergence of striking multistability. The authors of [39-41] did similar works by considering adjustable symmetry and nonlinearities in the dynamics of a simple jerk system, snap system, and jerk circuit, respectively. As far as symmetry breaking is concerned, the results obtained highlighted issues, such as the presence of parallel bifurcation branches, hysteresis, and coexisting multiple asymmetric attractors in the mentioned systems and circuits. These results are of particular practical interest given that perfect symmetry is not a practical reality.

Motivated by these results, we propose in this article a third-order chaotic system with adjustable symmetry, nonlinearity, and nontrivial equilibria as well. The novel system is a generalized form of the third-order autonomous Duffing-Holmes oscillator introduced by Lindberg et al. [42]. The novelty is brought by adding a parametric quadratic term to the cubic nonlinear term of the former system, resulting in a nonlinear function of the form $\varphi_{m}(x)=x-m x^{2}-x^{3}$. The quadratic term has been considered here because it models a possible imperfection that breaks the perfect symmetry of the original system. This modification can also be regarded as mathematical techniques to discover new nonlinear patterns. Historically, forced Duffing-Holmes system is one of the well-known simple nonautonomous second-order chaotic system [43, 44]. In 2009, Lindberg et al. [42] suggested an autonomous third-order Duffing-Holmes type system, with a smoother spectrum. In spite of the numerous studies recently devoted to uncovering multistability in nonlinear systems, no report has shown that the autonomous thirdorder Duffing-Holmes system suggested by Lindberg et al. has such striking behavior (to the best of the authors' knowledge). With the generalized third-order autonomous Duffing-Holmes system proposed in this work, our aim is twofold. Firstly, we show that the autonomous Duffing-Holmes system introduced by Lindberg et al. (which is a particular case of the generalized one) exhibits multistability. Secondly, we investigate the effects of adjustable symmetry, nonlinearity, and nontrivial equilibria on the dynamics previously obtained (with the particular case). Moreover, amplitude control and adaptive synchronization of the system are performed for promoting possible applications in engineering.

The remainder of this article is structured as follows. Section 2 is devoted to the description of the novel system and analytical examination of its basic properties. In Section 3 , the system is numerically analyzed using appropriate tools of nonlinear dynamics. In Section 4, an electronic analog calculator of the system is designed and PSpice simulation results are also presented. Sections 5 and 6 are about amplitude control and adaptive synchronization, respectively. Finally, conclusions are drawn in Section 7.

\section{System Description and Basic Properties}

2.1. System Description. The autonomous Duffing-Holmes system derived by Lindberg et al. [42] is as follows:

$$
\left\{\begin{array}{l}
\dot{x}=y, \\
\dot{y}=x-x^{3}+b y-k z, \\
\dot{z}=w_{f}(y-z),
\end{array}\right.
$$

where $b$ represents the damping coefficient, $k$ is the feedback coefficient, and $w_{f}$ is the cutoff frequency of the filter (see Ref. [42] for more details). The system under investigation is obtained by adding the parametric quadratic term $m x^{2}$ to the second equation of the autonomous Duffing-Holmes system (1), that is:

$$
\left\{\begin{array}{l}
\dot{x}=y \\
\dot{y}=x-x^{3}+b y-k z-m x^{2} \\
\dot{z}=w_{f}(y-z)
\end{array}\right.
$$

The quadratic nonlinearity coefficient $m$ is introduced to modify system's nonlinearity, symmetry, and equilibria as it will be shown later. Note that system (1) can always be obtained by setting $m=0$. Though this has been studied in [42], some complex behaviors such as coexistence of attractors were not revealed. With the generalized model (system (2)), we are going to revisit system (1) and investigate the case with $m \neq 0$.

2.2. Dissipation and Existence of Attractors. The presence of attractors in a system can be determined by calculating its divergence. The divergence of system (2) is given by 


$$
\Lambda=\frac{\partial \dot{x}}{\partial x}+\frac{\partial \dot{y}}{\partial y}+\frac{\partial \dot{z}}{\partial z}=-\left(w_{f}-b\right) .
$$

Given that $w_{f}$ and $b$ are strictly positive, the divergence of system (2) is strictly negative whenever $w_{f}>b$ (case considered in this work). This proves the existence of attractors because the volume elements contract after a unit of time. This contraction reduces a volume $V_{0}$ by a factor $e^{-\left(w_{f}-b\right) t}$. This means that each volume containing the trajectory of system (2) converges to zero when $t$ tends to infinity at an exponential rate of $\left(w_{f}-b\right)$. Therefore, all the orbits of system (2) are finally limited to a specific subset having a zero volume. Thus, the asymptotic movement settles on an attractor [45-49].

2.3. Symmetry. Symmetry is an interesting feature of dynamic systems. It tells us if the solutions are unique or even. System (2) is invariant to the transformation $(x, y, z) \Leftrightarrow(-x,-y,-z)$ when $m=0$. In this case, if $s(x, y, z)$ is a solution of the system for a set of parameter values, then $s(-x,-y,-z)$ is also a solution for the same values of parameters. This symmetry will be responsible for the appearance of the solutions in pair by polarity inversion of the initial conditions [50] and thus leads to the coexistence of attractors in the state space. However, the aforementioned symmetry disappears for $m \neq 0$.

2.4. Analysis of Fixed Points and Hopf Bifurcation. When studying the dynamics of a system, it is always interesting to analyze the stability of its fixed points. By setting the lefthand side of equation (2) equal to zero $(\dot{x}=\dot{y}=\dot{z}=0)$, we find that system (2) has three fixed points: one trivial equilibrium $p_{1}\left(x_{01}, y_{01}, z_{01}\right)=(0,0,0)$ and two nontrivial equilibria $p_{2,3}\left(x_{02,03}, y_{02,03}, z_{02,03}\right)=\left(\left(-m \pm \sqrt{m^{2}+4} / 2\right)\right.$, $0,0)$. It should be noted that coordinates of nontrivial equilibria depend on the parameter $m$; it is therefore possible to adjust their positions by acting on the parameter $m$. The Jacobian matrix of system (2) associated with any of these fixed points is given by the following expression:

$$
M_{J}=\left[\begin{array}{ccc}
0 & 1 & 0 \\
1-3 x^{2}-2 m x & b & -k \\
0 & w_{f} & -w_{f}
\end{array}\right] \text {. }
$$

The nature of equilibria can be found from the following characteristic equation $\left(\operatorname{det}\left(M_{J}-\lambda I_{3}\right)=0\right)$ :

$$
\begin{aligned}
\lambda^{3} & +\left(w_{f}-b\right) \lambda^{2}+\left(3 x^{2}+2 m x-b w_{f}+k w_{f}-1\right) \lambda \\
& +w_{f} x(3 x+2 m)-w_{f}=0,
\end{aligned}
$$

where $I_{3}$ denotes the $3 \times 3$ identity matrix. By applying Routh-Hurwitz stability criteria to characteristic equation (5), we obtain the following generalized stability conditions for the equilibrium points of system (2):

$$
\left\{\begin{array}{l}
\left(w_{f}-b\right)>0 \\
\left(w_{f}-b\right)\left(3 x^{2}+2 m x-b w_{f}+k w_{f}-1\right) \\
\quad-w_{f} x(3 x+2 m)+w_{f}>0, \\
w_{f}(x(3 x-2 m)-1)>0 .
\end{array}\right.
$$

For the trivial fixed point $p_{1}\left(x_{01}, y_{01}, z_{01}\right)=(0,0,0)$, we obtain the following stability conditions:

$$
\left\{\begin{array}{l}
w_{f}>b, \\
k>\left(1-\frac{1}{w_{f}\left(w_{f}-b\right)}\right) b \\
w_{f}<0,
\end{array}\right.
$$

while for the equilibrium points $P_{2,3}$, we have the following stability conditions:

$$
\left\{\begin{array}{l}
w_{f}>b \\
k>\frac{b\left(-w_{f}^{2}-3 x_{02,03}^{2}-2 m x_{02,03}+b w_{f}+1\right)}{w_{f}\left(-w_{f}+b\right)} \\
w_{f}>0 \\
m< \pm \frac{2}{15} \sqrt{15}
\end{array}\right.
$$

with

$$
x_{02,03}=\frac{-m \pm \sqrt{m^{2}+4}}{2} .
$$

Table 1 confirms relations (7) and (8), for different values of the parameters $w_{f}$, bet $m$.

Considering the change of variable $\lambda=i w_{0}\left(w_{0}>0\right)$ and substituting into (5), after separation of real part from imaginary part, we obtain relation (10) for the trivial point of equilibrium $\left(P_{1}\right)$ when $m=0$.

$$
\begin{aligned}
w_{\text {Hop } f} & =w_{0}=\sqrt{\frac{w_{f}}{b-w_{f}}}, \\
k_{c} & =\left(1-\frac{1}{w_{f}\left(w_{f}-b\right)}\right) b .
\end{aligned}
$$

Differentiation of equation (5) with respect to $k$ gives the following relation:

$$
\left.\operatorname{Real}\left(\frac{\partial \lambda}{\partial k}\right)\right|_{k=k_{c}}=\frac{-1}{2 w_{f}} \frac{1}{\left(\left(b-w_{f}\right)^{2}-1\right)^{2}+1} \neq 0, \quad \forall w_{f}>0
$$


TABLE 1: Stability of the equilibrium points $P_{1}, P_{2}$, and $P_{3}$ obtained from equations (7) and (8) for different values of $w_{f}, b, k$, and $m$.

\begin{tabular}{ccccccccc}
\hline \multirow{2}{*}{ Equilibrium points } & \multicolumn{3}{c}{ Parameter values } & & \multicolumn{2}{c}{ Eigenvalues } & $\lambda_{3}$ & Stability \\
& $w_{f}$ & $b$ & $k$ & $m$ & $\lambda_{1}$ & $\lambda_{2}$ & Stable \\
& -1 & -3 & -8 & - & $-0.2848+0.0000 \mathbf{i}$ & $-0.8576+1.6661 \mathbf{i}$ & $-0.8576-1.6661 \mathbf{i}$ & Stable \\
\multirow{2}{*}{$P_{1}$} & -2 & -4 & -7 & - & $-0.4668+0.0000 \mathbf{i}$ & $-0.7666+1.9227 \mathbf{i}$ & $-0.7666-1.9227 \mathbf{i}$ & Unstable \\
& 1 & 3 & 10 & - & $0.1761+0.0000 \mathbf{i}$ & $0.9120+2.2016 \mathbf{i}$ & $0.9120-2.2016 \mathbf{i}$ & Unstable \\
\hline \multirow{2}{*}{$P_{2}$} & 2 & 4 & 9 & - & $0.2329+0.0000 \mathbf{i}$ & $0.8836+2.7942 \mathbf{i}$ & $0.8836-2.7942 \mathbf{i}$ & Unstable \\
& 1 & 3 & 1.6696 & 0.6 & $-0.7163+0.0000 \mathbf{i}$ & $1.3582+0.5695 \mathbf{i}$ & $1.3582-0.5695 \mathrm{i}$ & Stable \\
\hline \multirow{2}{*}{$P_{3}$} & 3 & 1 & 2.2589 & 0.6 & $-1.5882+0.0000 \mathbf{i}$ & $-0.2059+3.3692 \mathbf{i}$ & $-0.2059-3.3692 \mathbf{i}$ & Unstable \\
& 2 & 4 & 2.1936 & -0.6 & $-1.4073+0.0000 \mathbf{i}$ & $1.7036+1.0422 \mathbf{i}$ & $1.7036-1.0422 \mathbf{i}$ & Utable \\
\hline
\end{tabular}

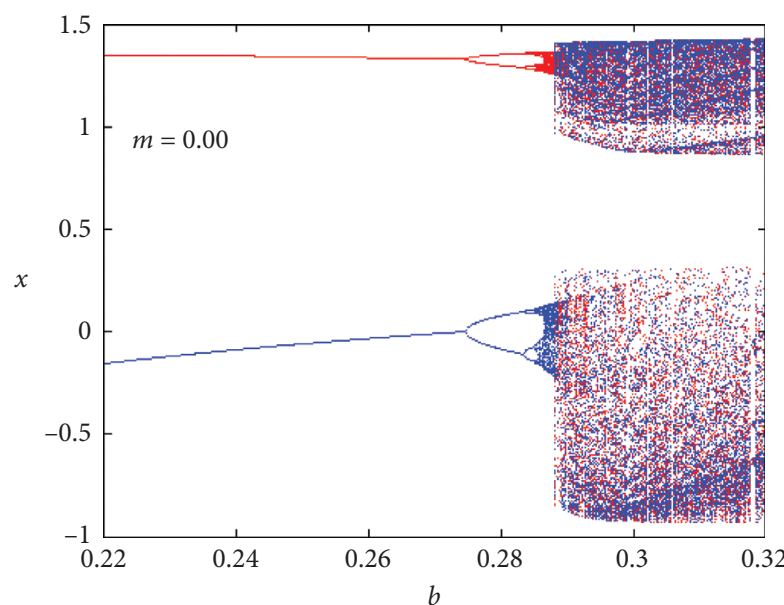

(a)

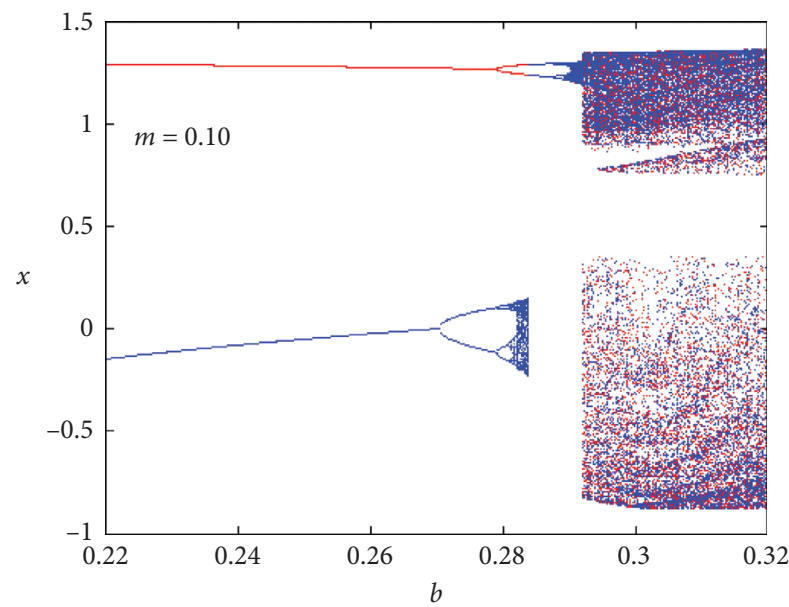

(c)

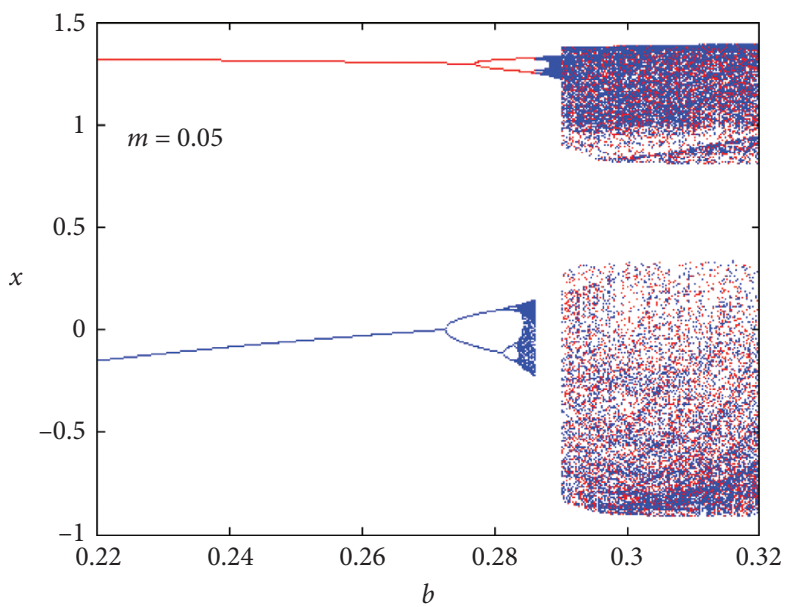

(b)

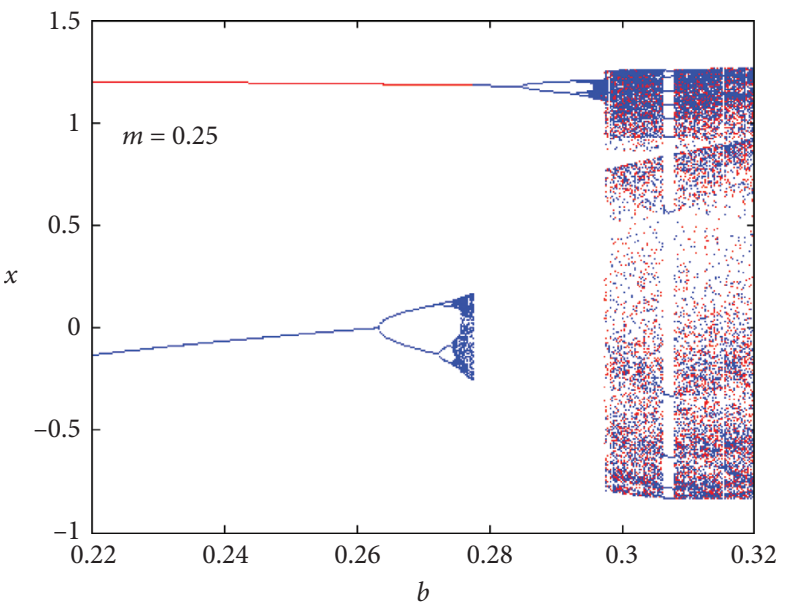

(d)

FIGURE 1: Bifurcation diagrams as a function of the parameter b showing destruction of the symmetry of the system for some values of $m$ : (a) the symmetric system for $m=0.00$; (b-d) destruction of the system's symmetry for $m=0.05, m=0.10$, and $m=0.25$, respectively. The initial conditions are $(x(0), y(0), z(0))=(0.25,0,0)$ and $(-0.25,0,0)$ for the traces in red and blue, respectively.

Equation (10) defines the frequency of stable oscillations as well as the critical value of $k$ corresponding to the Hopf bifurcation of system (2). From relation (11), note that the transversality condition is always satisfied provided that $w_{f}$ is a strictly positive parameter.

\section{Numerical Investigations}

3.1. Methodology. In this section, we do a thorough analysis of the dynamics of system (2) using numerical tools such as bifurcation diagrams, maximum Lyapunov exponent, 
Poincaré section, phase portraits, time series, cross section of the basin of attraction, and so on. Bifurcation diagrams are obtained from the local maxima of one of the state variables for different values of the control parameters. Maximum Lyapunov exponents are computed using Wolf ${ }^{\circ}$ algorithm [51]. In fact, the Maximum Lyapunov exponent makes it possible to estimate the rate of convergence or divergence between two close trajectories. Thus, if the maximum Lyapunov exponent is (i) positive, the system is considered as chaotic because for a small perturbation, its trajectory diverges (this implies the occurrence of a strange attractor in phase space); (ii) negative, the system is considered stable because for all perturbations, it returns to its equilibrium point; (iii) null, the system is considered periodic or quasiperiodic.

All the numerical integrations are based on the fourthorder Runge-Kutta algorithm with an integration step $h=5.10^{-3}$, for better precision.

\subsection{Coexistence of Bifurcations and Multiple Attractors}

3.2.1. Considering $b$ as a Control Parameter with $m=0$. In this section, we consider $b$ as a control parameter and $m$ is equal to zero. The dynamics obtained is that represented by the bifurcation diagrams of Figure 1(a), carried out in the ascending direction for $0.22 \leq b \leq 0.32, w_{f}=0.5$, and $k=1.6$. This figure presents a very interesting phenomenon which is the coexistence of two symmetric bifurcation diagrams. This coexistence is highlighted through the superposition of two diagrams (red and blue) obtained by symmetrically changing the initial conditions $((0.25,0,0) \Leftrightarrow(-0.25,0,0))$. They are in good agreement with the corresponding graphs of Lyapunov exponents as shown in Figure 2(a). Figure 3 shows some 3D phase portraits obtained for different values of the control parameter with initial conditions as indicated. Figures 4(a)4(d) show a symmetric chaotic attractor, its time series, its frequency spectrum, and its Poincaré section (these representations are consistent with the chaotic behavior), respectively, for $b=0.32$. All these confirm the symmetric nature of the dynamic of system (2).

3.2.2. Considering $b$ as a Control Parameter with $m \neq 0$. In this section, we still consider $b$ as a control parameter but $m \neq 0.0$. With $0.22 \leq b \leq 0.32, w_{f}=0.5$, and $k=1.6$, bifurcation diagrams shown in Figures 1(b)-1(d) are plotted for $m$ equal to $0.05,0.10$, and 0.25 , respectively. Let us recall that red color is used for $\left(x_{0}, y_{0}, z_{0}\right)=(0.25,0,0)$ and blue color is used for $\left(x_{0}, y_{0}, z_{0}\right)=(-0.25,0,0)$. Comparing Figures 1(b)-1(d) with Figure 1(a), we can observe that bifurcation diagrams in red are no more symmetric to their counterpart (in blue). There is a gap (discontinuity) in the blue bifurcation diagrams (Figures 1(b)-1(d)), which increases as $m$ is increased. This observation is caused by a shift in equilibrium points (see Table 2) and implies the existence of coexisting attractors. The coexistence of a period-2 limit cycle with a period-3 limit cycle (Figure 5(c)) and a chaotic attractor with a periodic attractor (Figure 5(d)), for instance, is revealed in Figure 5. Figures 6(a)-6(d) represent an asymmetric chaotic attractor, its time series, its frequency spectrum, and its Poincaré section (these representations are specific to chaotic attractors), respectively, for $b=0.32$. The aforementioned results indicated an increase in system's complexity when $m \neq 0$.

3.2.3. Considering $k$ as a Control Parameter with $m=0$. Considering $k$ as a control parameter with $m$ equal to zero, the dynamics of system (2) is governed by the superimposed bifurcation diagrams and Lyapunov exponents of Figures $7(a)$ and $8(a)$, respectively. These diagrams are obtained by varying the control parameter (downward) in the range $2 \leq k \leq 3$ for initial conditions of opposite signs $( \pm 0.75,0,0)$ with $w_{f}=0.5$ and $b=0.41$. Figure 9 shows an enlargement of the diagram of Figure 7 (a) (in red) in the intervals $2.6575 \leq k \leq 2.68$ (Figure 9(a)) and $2.671 \leq k \leq 2.672$ (Figure 9(b)) illustrating parallel branches. They show the coexistence of four (two periodic and two chaotic) and six attractors occurring in symmetric pairs. Figures 10 and 11 illustrate the $2 \mathrm{D}$ representations of these different attractors in the phase space. These attractors are obtained for the values of $k=2.667$ and 2.6714, respectively. The different values of the initial conditions $\left(x_{0}, y_{0}, z_{0}\right)$ are as follows: for the coexistence of the four attractors-period-1 $( \pm 0.12$, $0,0)$ and chaotic spiral $( \pm 0.39,0,0)$; for the coexistence of the six attractors $-n$-finite periods $( \pm 0.28,0,0)$, period$6( \pm 0.06,0,0)$, and period-1 $( \pm 0.108,0,0)$. These multistabilities are well illustrated on the cross sections of the basins represented in Figures 12(a)-12(d), where we can observe several regions distinguished by different colors that correspond to each attractor.

3.2.4. Considering $k$ as a Control Parameter with $m \neq 0$. Keeping $k(2 \leq k \leq 3)$ as the control parameter, with $m \neq 0(m=0.10,0.125), w_{f}=0.5$, and $b=0.41$, we obtain the asymmetric bifurcation diagrams of Figures $7(\mathrm{~b})$ and 7(c), which govern the evolution of system (2) for $m=0.10$ and 0.125 , respectively. These diagrams are obtained for the initial conditions $( \pm 0.75,0,0)$. The asymmetry observed in these diagrams is due to the destruction of the system's symmetry. This is caused by a nonsymmetric displacement of nontrivial equilibrium points with respect to the values of $m$ (see Table 2). This can also be observed on the corresponding Lyapunov exponents diagrams of Figures $8(\mathrm{~b})$ and $8(\mathrm{c})$.

3.3. Two Parameters and Lyapunov Exponents. The changes that occur in chaotic dynamic systems may not be only related to small differences of initial conditions but also rely on the variation of parameters to which the system is sensitive. Figure 13 shows an interesting way to represent the behavior of system (2) according to its parameters 


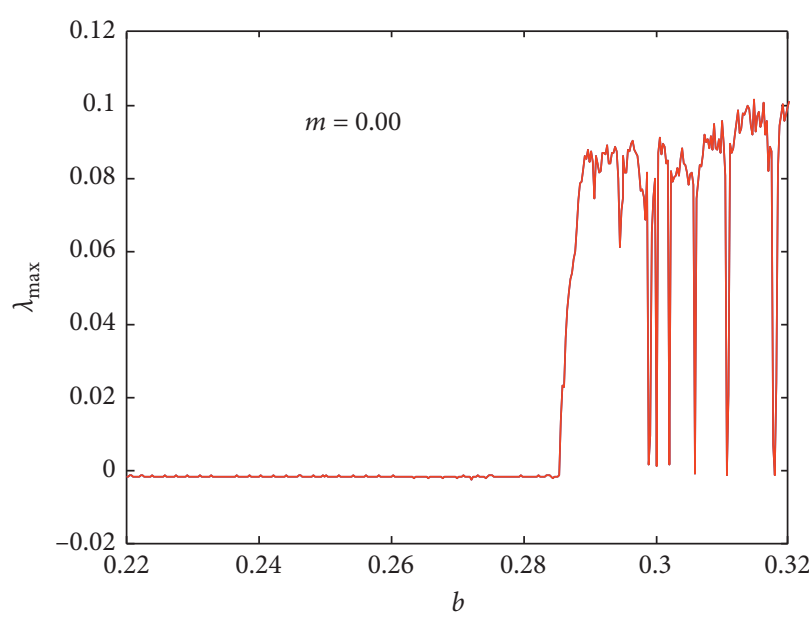

(a)

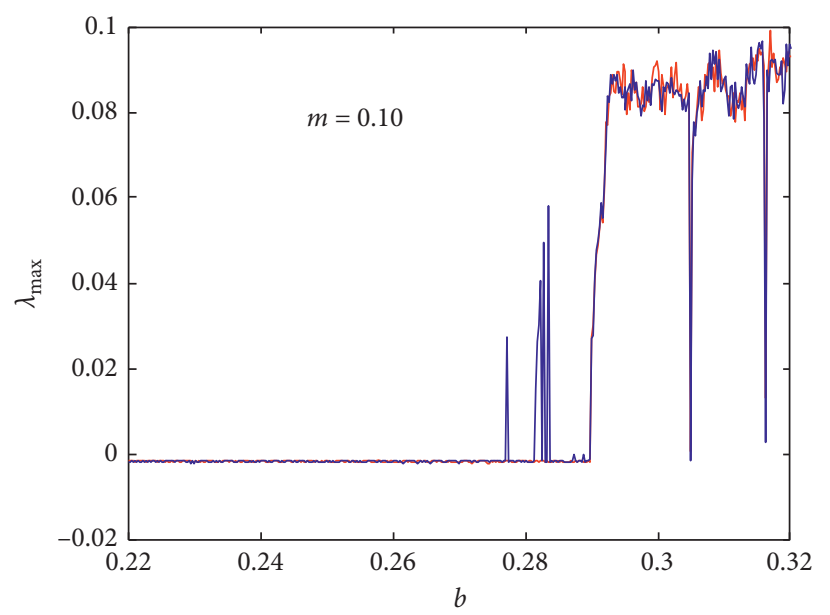

(c)

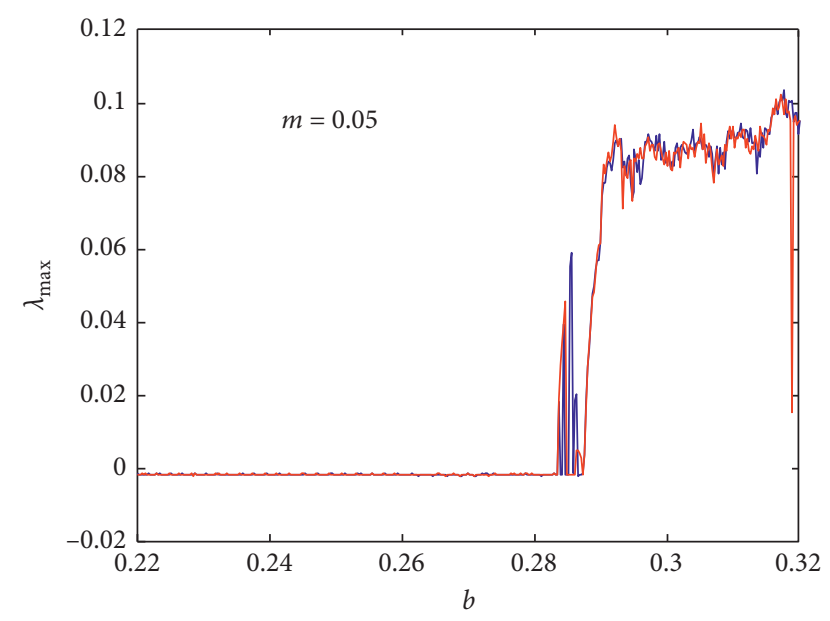

(b)

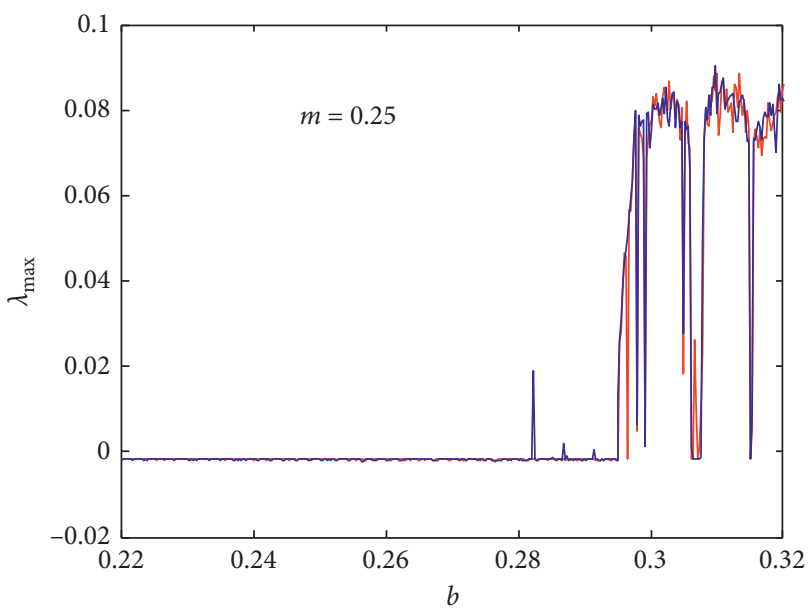

(d)

Figure 2: Diagrams of the maximum Lyapunov exponents, corresponding to the bifurcation diagrams of Figure 1 . This figure shows the impact of the value of $m$ on the symmetry of system (2) considering $b$ as a control parameter.

$\left(b, k\right.$, and $\left.w_{f}\right)$ through the maximum Lyapunov exponents $\left(\lambda_{\max }\right)[52,53]$. In this figure, we can appreciate behavioral changes marked by transitions, from periodic zones (where $\lambda_{\max }<0$ ) in blue to chaotic zones (where $\lambda_{\max }>0$ ) in red (see Figures 13(a) and 13(b)). This way of presenting the dynamics of the system (2) thus makes it possible to prove the existence of chaotic solution for a combination of the values of its parameters.

\section{Circuit Design and PSpice Simulations}

The theoretical and numerical predictions previously obtained are verified in this section using an electronic analog of system (2). The circuit diagram of the proposed electronic analog computer is shown in Figure 14. It involves three integrators, an inverter, a subtractor, and two multipliers which are at the origin of the nonlinear terms.

Using Kirchhoff's laws, equations governing the circuit can be obtained as

$$
\left\{\begin{array}{l}
\frac{1}{R C} \frac{\mathrm{d} x}{\mathrm{~d} \tau}=\frac{1}{R C} y, \\
\frac{1}{R C} \frac{\mathrm{d} y}{d \tau}=\frac{1}{R C} y-\frac{1}{R C} x^{3}+\frac{R_{b}}{R C} y-\frac{R_{k}}{R C} z-\frac{R_{m}}{R C} x^{2}, \\
\frac{1}{R C} \frac{\mathrm{d} z}{\mathrm{~d} \tau}=\frac{R_{w_{f}}}{R C}(y-z),
\end{array}\right.
$$

with the following considerations:

$$
\begin{aligned}
& x=X, \\
& y=Y, \\
& z=Z, \\
& \tau=\frac{t}{R C} .
\end{aligned}
$$

Equation (12) becomes 


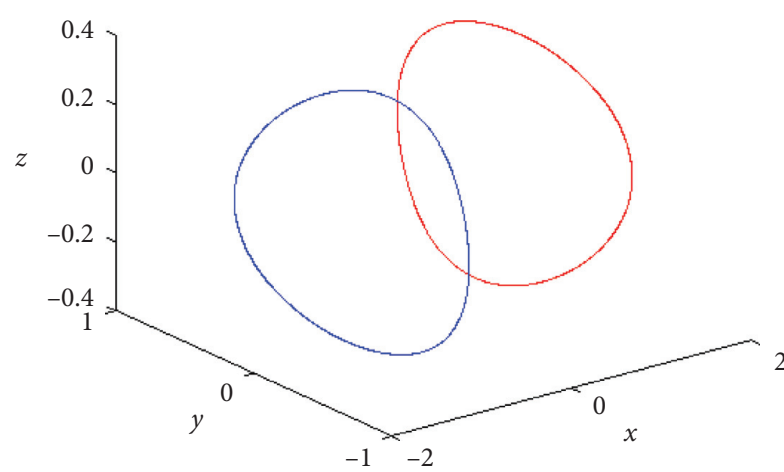

(a)

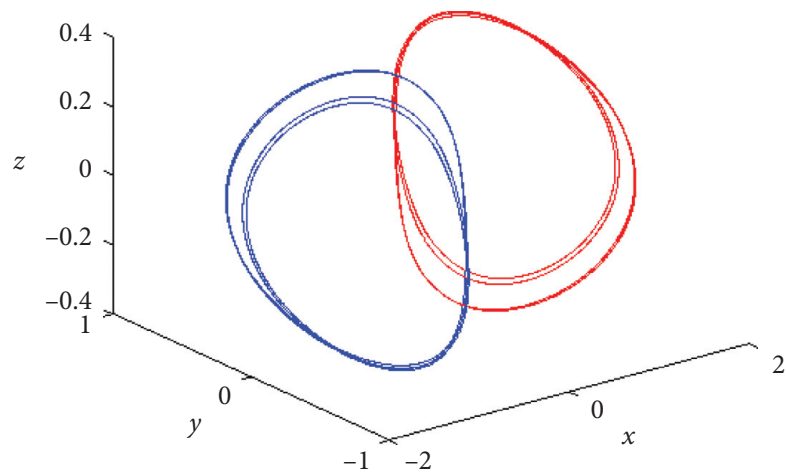

(c)

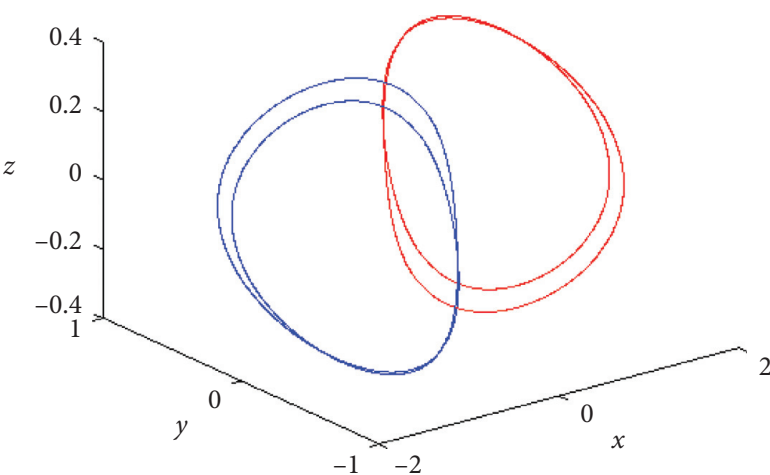

(b)

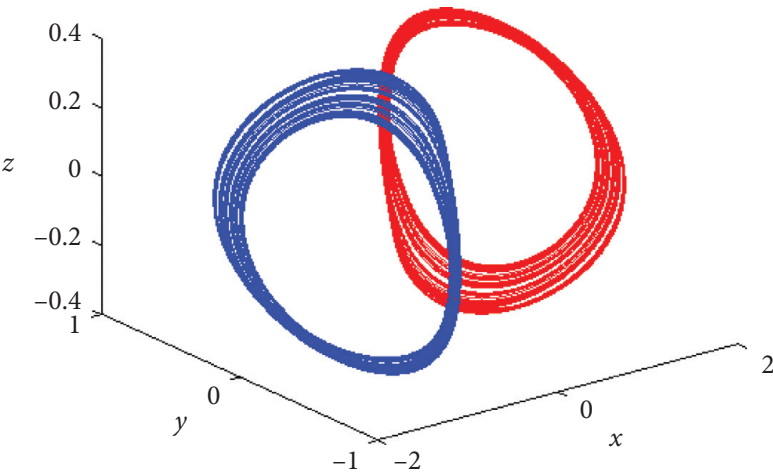

(d)

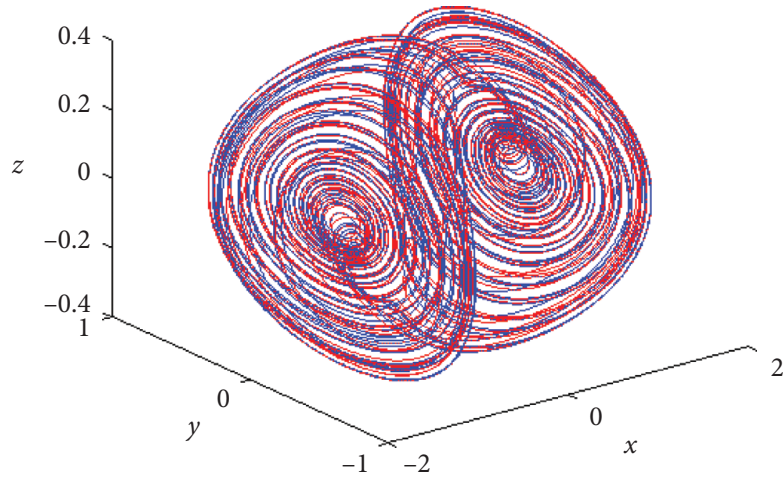

(e)

Figure 3: Three-dimensional representations $(x-y-z)$ of trajectories in phase space, at different points of the bifurcation diagram of the symmetry system (Figure 1(a)) for different values of $b$ : (a) cycle of period-1 for $b=0.22$, (b) cycle of period-2 for $b=0.28$, (c) cycle of period-4 for $b=0.284$, and (d, e) single and double chaotic bands for $b=0.288$ and $b=0.3$, respectively. The initial conditions are $(x(0), y(0), z(0))=(0.25,0,0)$ and $(-0.25,0,0)$ for the curves in red and blue, respectively.

$$
\left\{\begin{array}{lr}
\frac{d X}{\mathrm{~d} t}=\frac{1}{R C_{1}} Y, & b=\frac{R_{b}}{R} \\
\frac{d Y}{\mathrm{~d} t}=\frac{1}{R C_{2}} X-\frac{1}{R C_{2}} X^{3}+\frac{R_{b}}{R C_{2}} Y-\frac{R_{k}}{R C_{2}} Z-\frac{R_{m}}{R C_{2}} X^{2}, & k=\frac{R_{k}}{R} \\
\frac{d Z}{\mathrm{~d} t}=\frac{R_{w_{f}}}{R C_{2}}(Y-Z) . & w_{f}=\frac{R_{w_{f}}}{R}, \\
\text { Systems (14) and (2) are equivalent for } & (14)
\end{array}\right.
$$




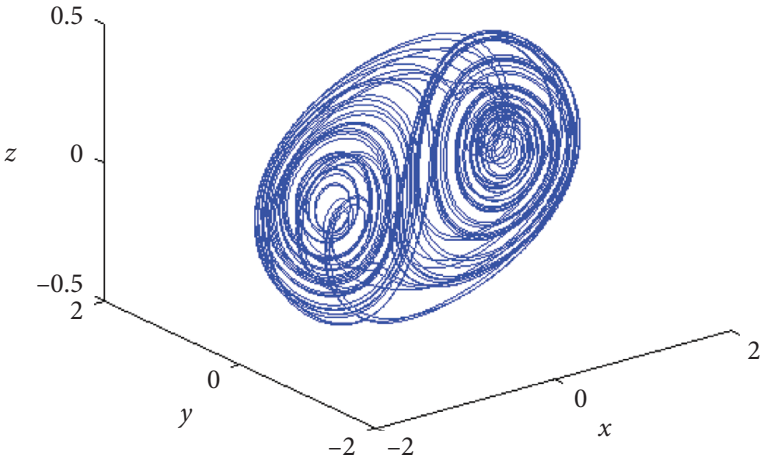

(a)

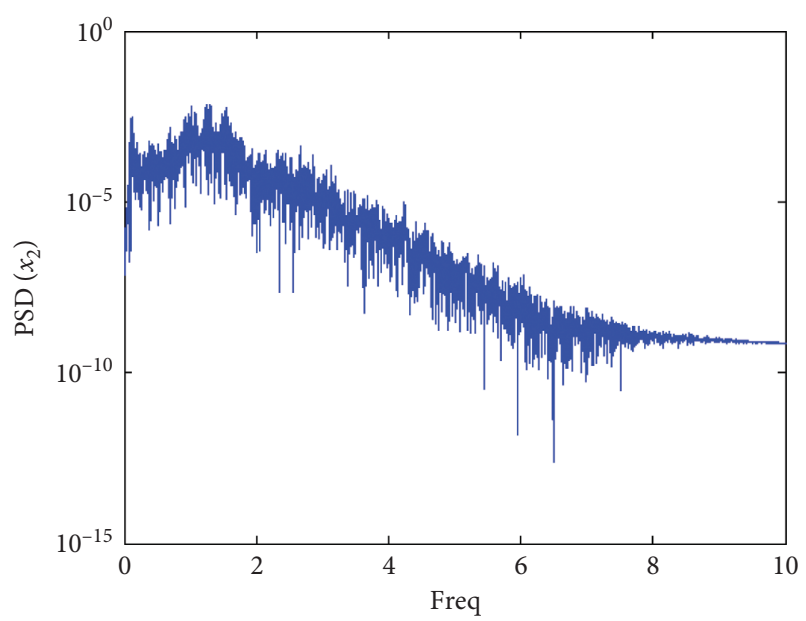

(c)

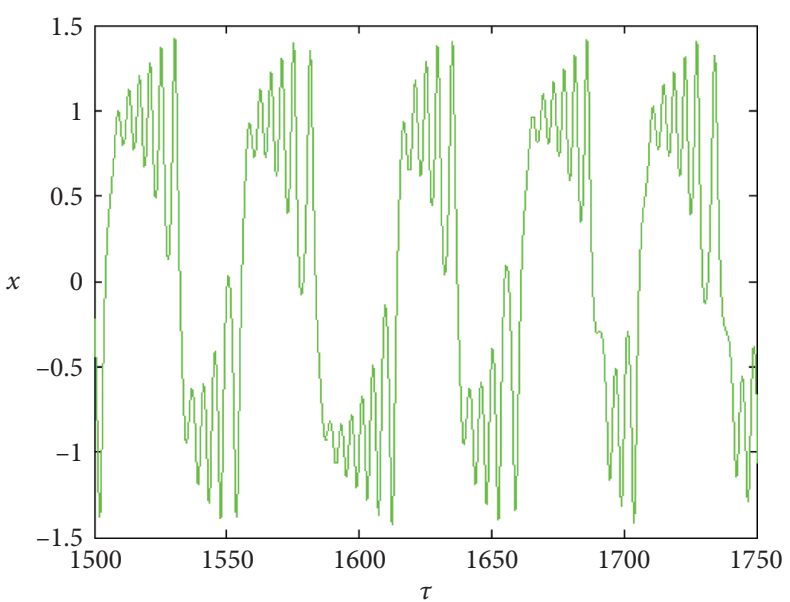

(b)

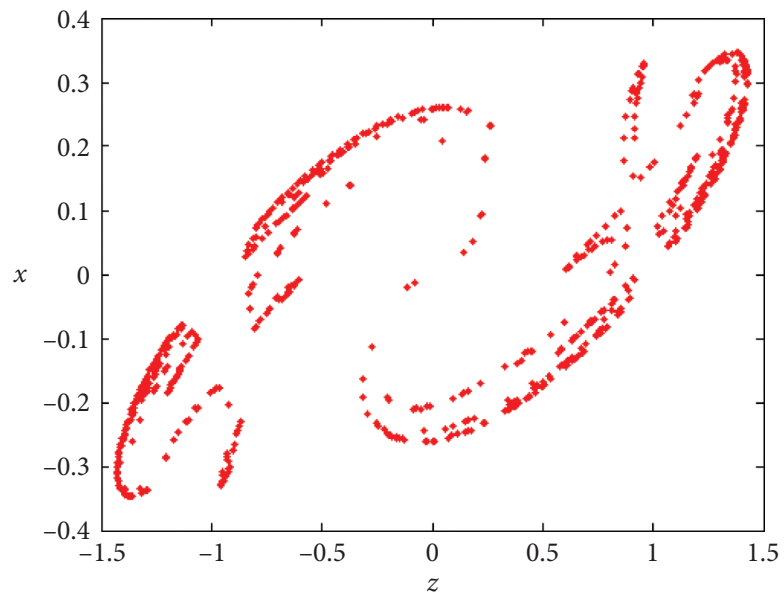

(d)

Figure 4: Three-dimensional portrait (a), time series (b), frequency spectrum (c), and Poincaré section (d), confirming symmetric chaotic behavior of the system for $m=0.00$ and $b=0.32$. The other parameters are specified in the text.

TABLE 2: Modifications of nontrivial equilibrium points for different values of $m$.

\begin{tabular}{lcr}
\hline Values of $m$ & \multicolumn{2}{c}{ Nontrivial equilibrium points } \\
\hline 0.00 & $(1,0,0)$ & $(-1,0,0)$ \\
0.05 & $(0.9753,0,0)$ & $(-1.0253,0,0)$ \\
0.10 & $(0.9512,0,0)$ & $(-1.0512,0,0)$ \\
0.125 & $(0.9395,0,0)$ & $(-1.0645,0,0)$ \\
0.25 & $(0.8828,0,0)$ & $(-1.1328,0,0)$ \\
\hline
\end{tabular}

Let us carry out analog simulations of the circuit's dynamics in PSpice. For these simulations, the following configurations of the transient analysis are adopted. Print step: 200 ns; final step: $500 \mathrm{~ms}$; no-print delay: $480 \mathrm{~ms}$; step ceiling: $4 \mu \mathrm{s}$. For electronic components, $C_{1}=C_{2}=C_{3}=10 \mathrm{nF}$ and $R=10 \mathrm{k} \Omega$ while $R_{b}, R_{k}, R_{m}$, and $R_{w_{f}}$ are tunable. The different portraits presented in Figure 14 correspond to the transitions to chaos of the symmetric system obtained by fixing
$R_{m}=1 \mathrm{M} \Omega, R_{w_{f}}=20 \mathrm{k} \Omega$, and $R_{k}=6.25 \mathrm{k} \Omega$ and by varying $R_{b}$ (see Table 3 ) for the initial conditions $(X(0), Y(0), Z(0))=$ $( \pm 0.25 \mathrm{~V}, 0 \mathrm{~V}, 0 \mathrm{~V})$. We can observe similarities between phase portraits of Figure 15 with those of Figure 3 obtained from the bifurcation diagram of the symmetric system (Figure 1(a)). Figure 16 shows the transitions of the asymmetric system to chaos obtained for $R_{m}=200 \mathrm{k} \Omega, R_{w_{f}}=20 \mathrm{k} \Omega, R_{k}=$ $6.25 \mathrm{k} \Omega$ by varying $R_{b}$ (see Table 3 ) with the initial conditions 


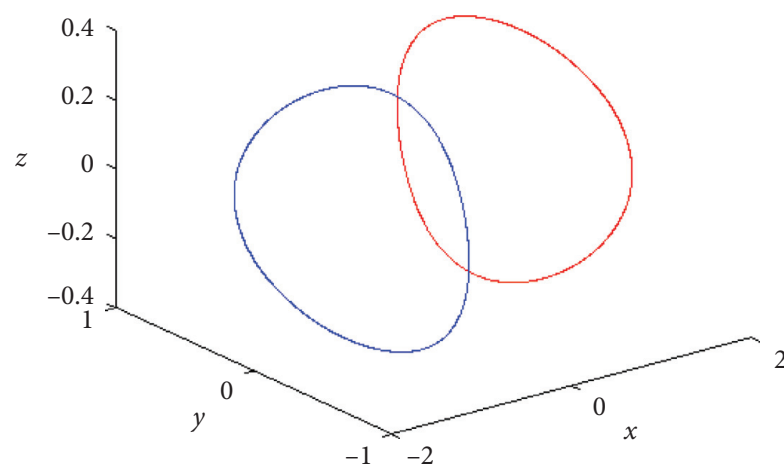

(a)

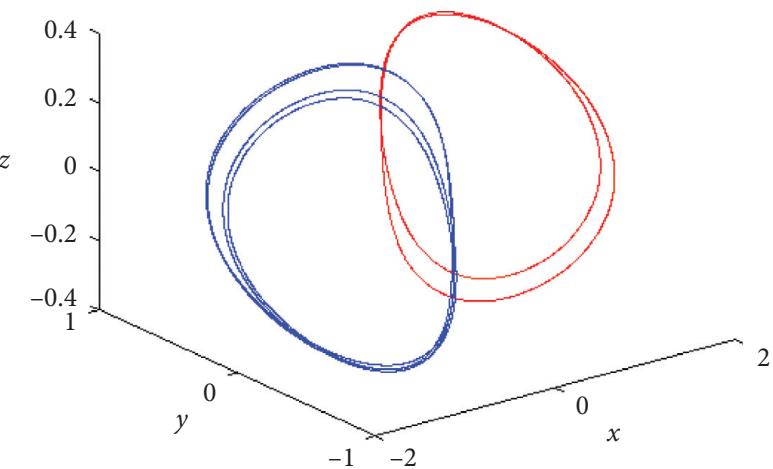

(c)

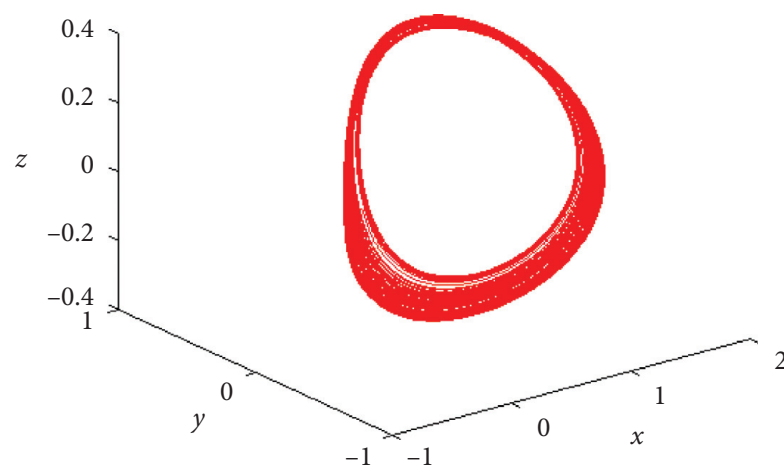

(e)

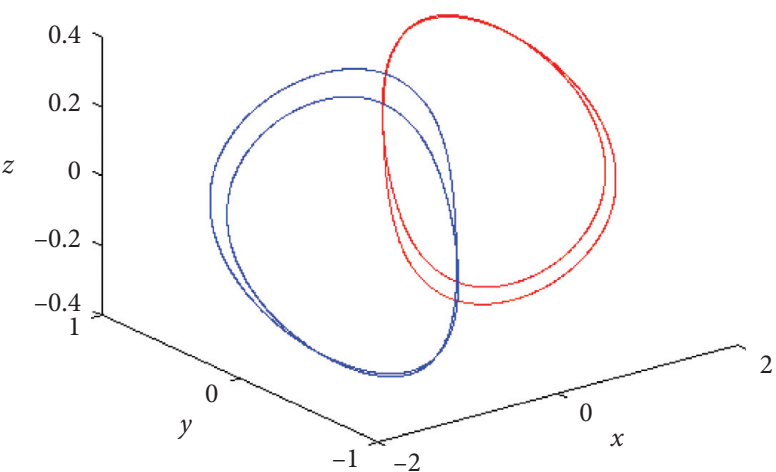

(b)

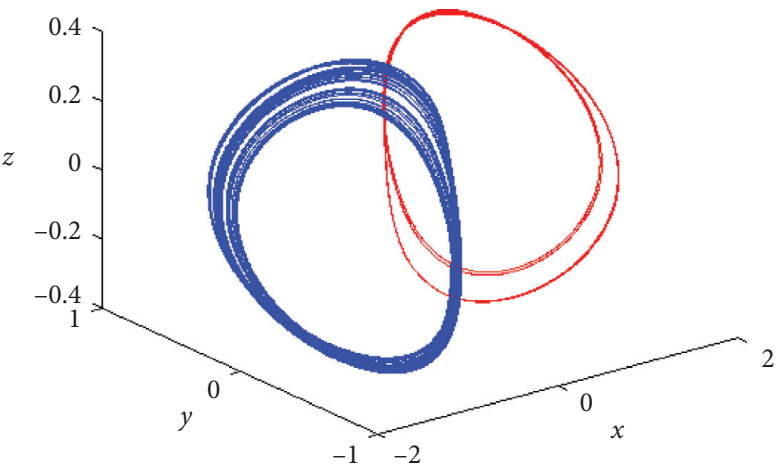

(d)

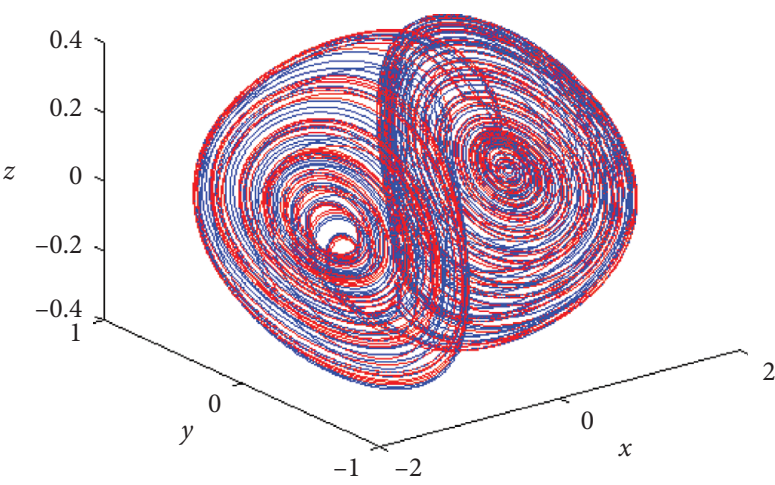

(f)

FiguRE 5: Three-dimensional representations $(x-y-z)$ of trajectories in the phase space, at different points of the bifurcation diagram of the asymmetry system (Figure 2(a)), for different values of $b$ with $m=0.05$ : (a, b) cycle of period- 1 for $b=0.22$ and cycle of period-2 for $b=0.25$; (c) cycles of period- 4 and period- 2 for $b=0.2824$; (d) single-band chaotic attractor with cycle of period- 4 for $b=0.2856$; (e, $\mathrm{f}$ ) single and chaotic dual-band attractor for $b=0.2892$ and $b=0.3$, respectively. The initial conditions are $(x(0), y(0), z(0))=(0.25,0,0)$ and $(-0.25,0,0)$ for the traces in red and blue, respectively.

$(X(0), Y(0), Z(0))=( \pm 0.15 \mathrm{~V}, 0 \mathrm{~V}, 0 \mathrm{~V})$. These transitions are similar to those of Figure 5 obtained by numerical integrations at different points of the bifurcation diagram of the asymmetric system (Figure 1(b)). The coexistence of the four and six attractors (see Figures 17 and 18) was obtained for $R_{k}=$ $3.746 \mathrm{k} \Omega$ and $R_{k}=3.745 \mathrm{k} \Omega$, respectively (see Table 3 ), with $R_{m}=1 \mathrm{M} \Omega, R_{w_{f}}=20 \mathrm{k} \Omega, R_{b}=24.39 \mathrm{k} \Omega$. Concerning the values of the initial conditions $(X(0), Y(0), Z(0))$, they are $( \pm 0.13 \mathrm{~V}, 0 \mathrm{~V}, 0 \mathrm{~V})$ and $( \pm 0.15 \mathrm{~V}, 0 \mathrm{~V}, 0 \mathrm{~V})$ for the four attractors and $( \pm 0.11 \mathrm{~V}, 0 \mathrm{~V}, 0 \mathrm{~V}),( \pm 0.25 \mathrm{~V}, 0 \mathrm{~V}, 0 \mathrm{~V})$, $(0.17 \mathrm{~V}, 0 \mathrm{~V}, 0 \mathrm{~V})$, and $(-0.88 \mathrm{~V}, 0 \mathrm{~V}, 0 \mathrm{~V})$ for the six attractors.

\section{Total Amplitude Control (TAC)}

Amplitude control of chaotic signals is important in engineering applications where the amplitude is desired for signal generation and transmission [54-58]. It makes it possible to get the attractor large or small by changing one or more variables in a range, without changing the dynamic and topological properties of the attractor [59]. The amplitude control can be total (TAC), in this case all variables are controlled in a linked way. When all the variables are controlled separately, we speak of composite amplitude control (CAC). In the case where only the amplitude of one 


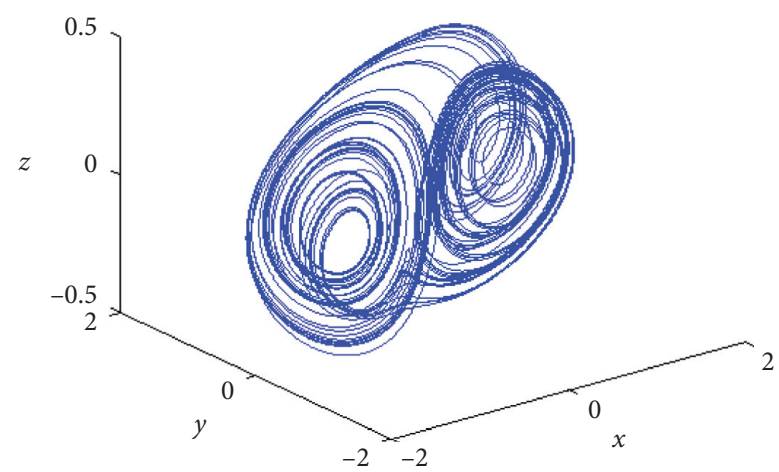

(a)

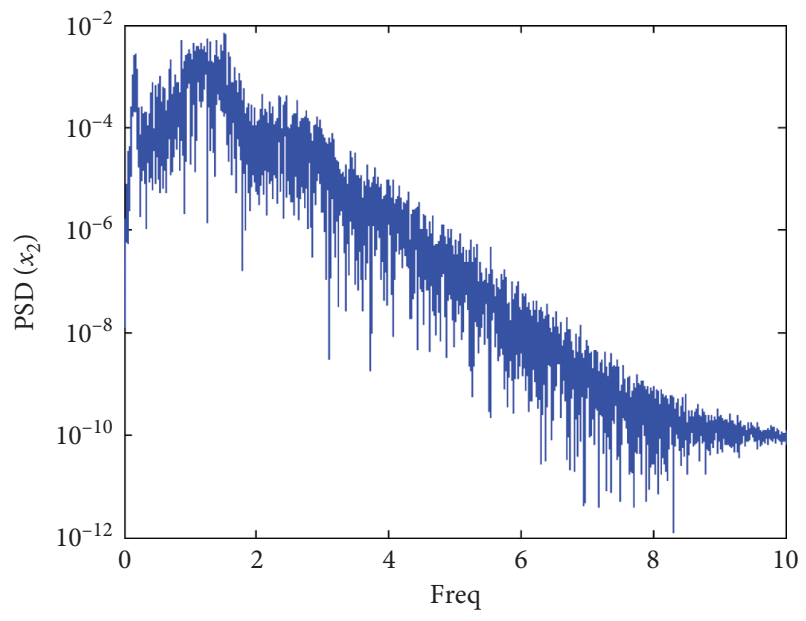

(c)

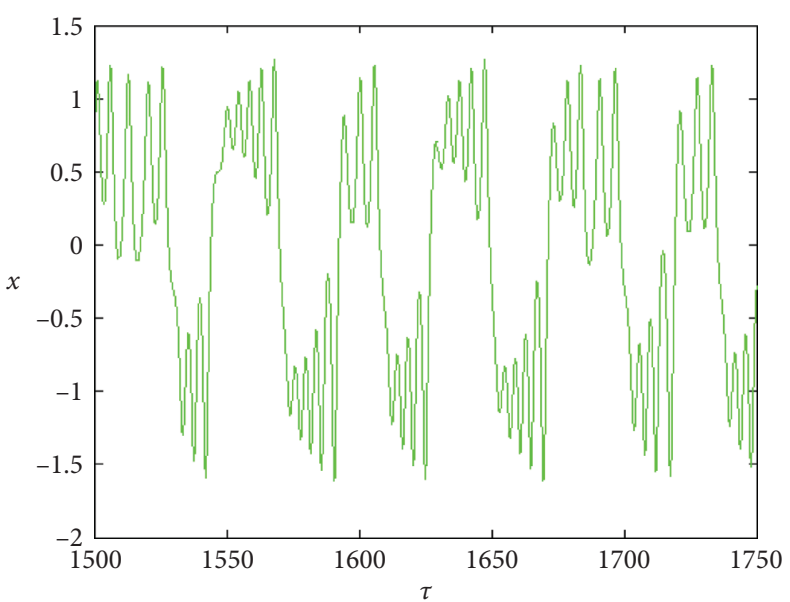

(b)

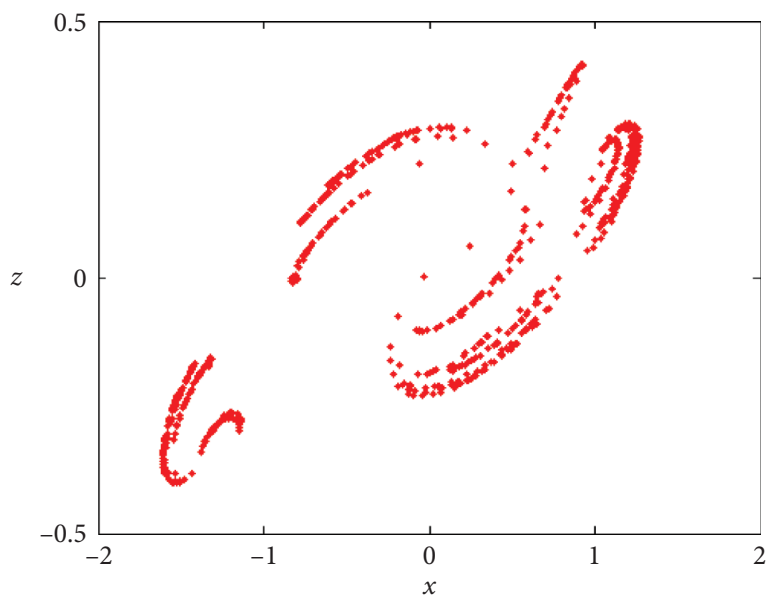

(d)

Figure 6: Three-dimensional portrait (a), time series (b), frequency spectrum (c), and Poincaré section (d), confirming asymmetric chaotic behavior of the system for $m=0.25$ and $b=0.32$. The other parameters are specified in the text.

or of $n-1(n \geq 3)$ variables is controlled, it is a partial amplitude control (PAC) [56, 59].

Referring to [56, 59-61], it is possible to perform a total amplitude control (TAC) of system (2) by introducing an amplitude control function $f(r)$ on the cubic term, which leads to the following system:

$$
\left\{\begin{array}{l}
\dot{x}=y, \\
\dot{y}=x-\frac{1}{f(r)} x^{3}+b y-k z-m x^{2}, \\
\dot{z}=w_{f}(y-z)
\end{array}\right.
$$

The function $f(r)$ makes it possible to control the amplitudes of the variables $x, y$, and $z$ according to $1 / \sqrt{f(r)}(f(r)>0)$.

Proof. Let: $u=x / \sqrt{f(r)}, v=y / \sqrt{f(r)}$ and $w=z / \sqrt{f(r) \text {. }}$ The resulting system (17) considering $m=0$ is identical to system (2):

$$
\left\{\begin{array}{l}
\dot{u}=v \\
\dot{v}=u-u^{3}+b v-k w-m \sqrt{f(r)} u^{2} \\
\dot{w}=w_{f}(v-w)
\end{array}\right.
$$

So, the function $f(r)$ controls all the amplitudes according to $1 / \sqrt{f(r)}$. Consider $f(r)=1 / r(r>0)$, with $r$ being the total amplitude control parameter. By acting on the amplitude control parameter $r(r>0)$, the variables are modified while leaving the dynamics of the attractor unchanged. Figure 19(a) shows the reductions in the amplitudes of the $x, y$, and $z$ variables of the dual-band chaotic attractor when increasing the control parameter $r$. Figure 19(b) shows the largest Lyapunov exponent diagram, which remains unchanged when the control parameter varies, which justifies the invariance of the properties of the attractor during amplitude control. The phase portraits of this dual-band chaotic attractor are shown in Figure 20 in the $(x-y),(x-z)$, and $(y-z)$ planes for the discrete values of $r$. These representations come to validate the total 


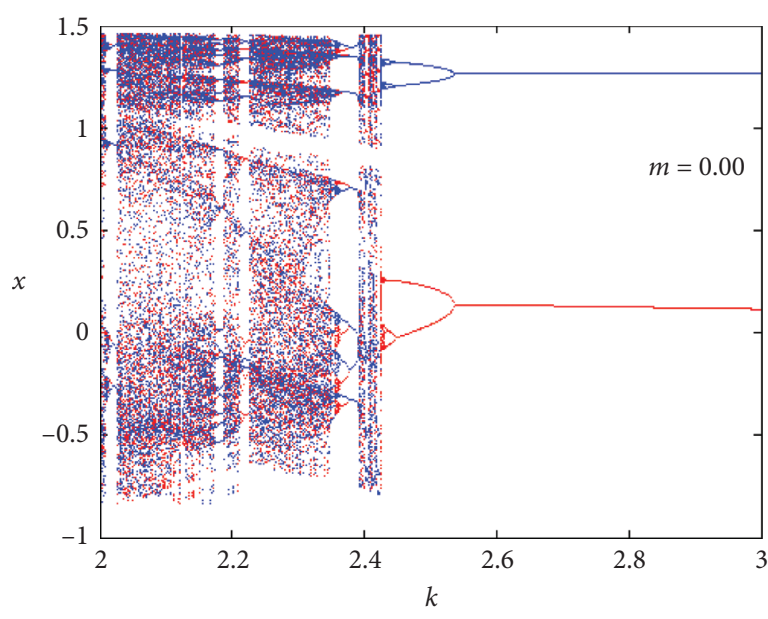

(a)

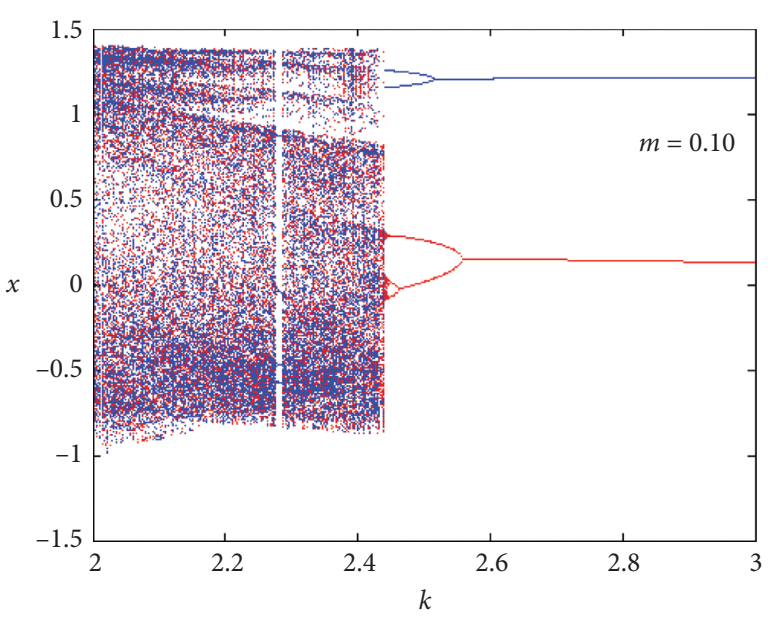

(b)

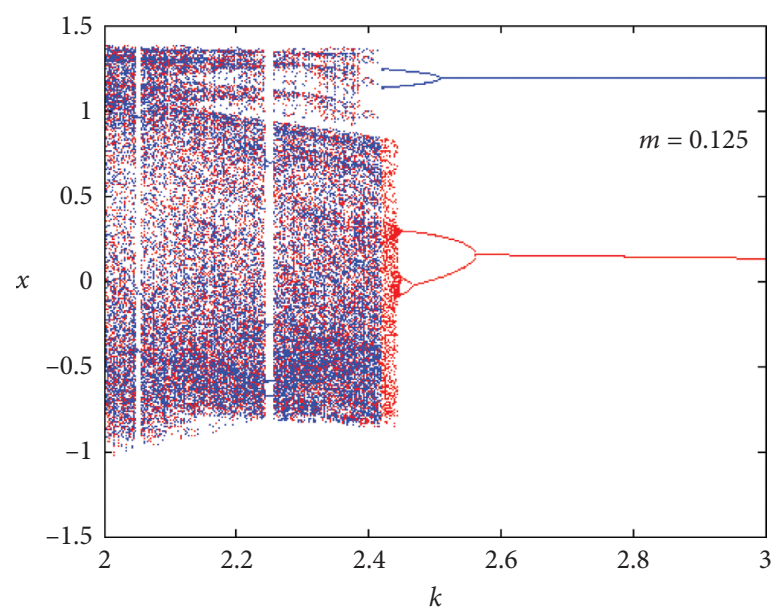

(c)

FiguRE 7: Bifurcation diagrams as function of the parameter $k$, showing destruction of the symmetry of the system for some values of $m$ : (a) the symmetric system for $m=0$; (b, c) destruction of the system symmetry for $m=0.10$ and $m=0.125$, respectively. The initial conditions are $(x(0), y(0), z(0))=(0.75,0,0)$ and $(-0.75,0,0)$ for the traces in blue and red, respectively.

amplitude control (TAC) because the amplitudes of the three variables are reduced without altering the dynamic and topological properties of the attractor. This control can be achieved by introducing a potentiometer in the electronic circuit. This is convenient or beneficial in the context of secure communication as well as in various fields of information processing in engineering [54-58].

\section{Adaptive Synchronization}

The importance of synchronizing chaotic and/or hyperchaotic systems could be its application in areas of engineering such as secure communication [62-66]. To date, there are several synchronization schemes that have already been developed with notable applications [67-71]. Among these, one of the best known methods is the adaptive method, which we will use in this section.
Consider the master and slave subsystems described by expressions (18) and (19), respectively:

$$
\begin{aligned}
& \left\{\begin{array}{l}
\dot{x}_{1}=y_{1}, \\
\dot{y}_{1}=x_{1}-x_{1}^{3}-m x_{1}^{2}+b y_{1}-k z_{1}, \\
\dot{z}_{1}=w_{f}\left(y_{1}-z_{1}\right),
\end{array}\right. \\
& \left\{\begin{array}{l}
\dot{x}_{2}=y_{2}+u_{1}, \\
\dot{y}_{2}=x_{2}-x_{2}^{3}-m x_{2}^{2}+b y_{2}-k z_{2}+u_{2}, \\
\dot{z}_{2}=w_{f}\left(y_{2}-z_{2}\right)+u_{3},
\end{array}\right.
\end{aligned}
$$

where $\left(x_{1}, y_{1}, z_{1}\right)$ and $\left(x_{2}, y_{2}, z_{2}\right)$ represent the state variables of the master and slave, respectively. $u_{i}(i=1,2,3)$ are the nonlinear controllers to choose so that the subsystems (18) and (19) synchronize.

The synchronization errors between (18) and (19) are given by 


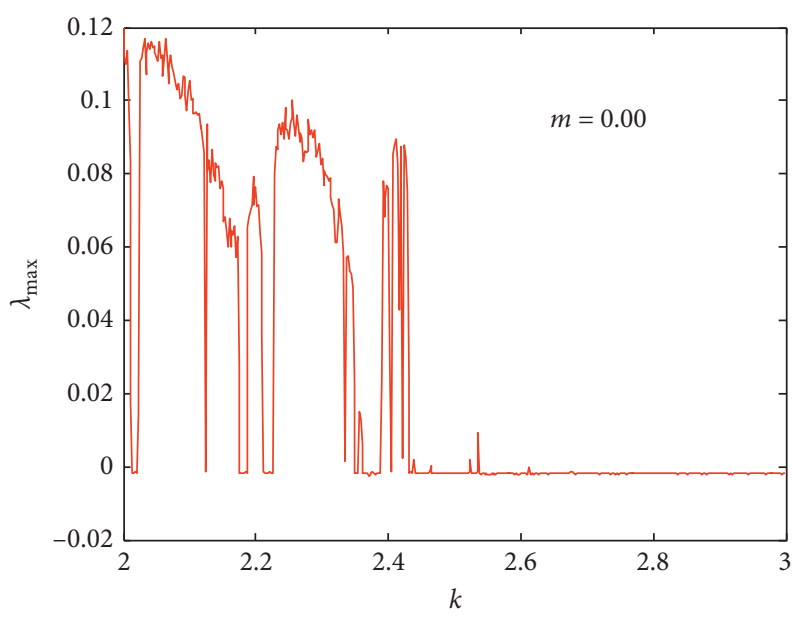

(a)

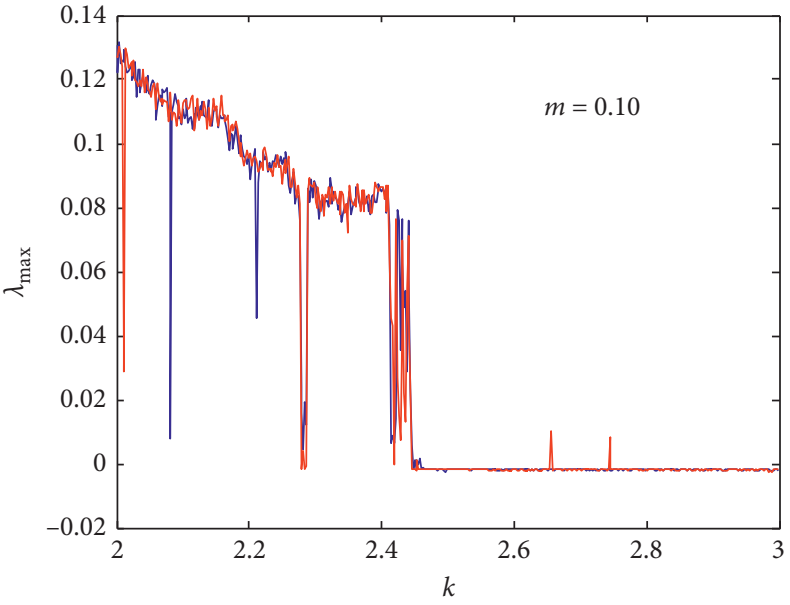

(b)

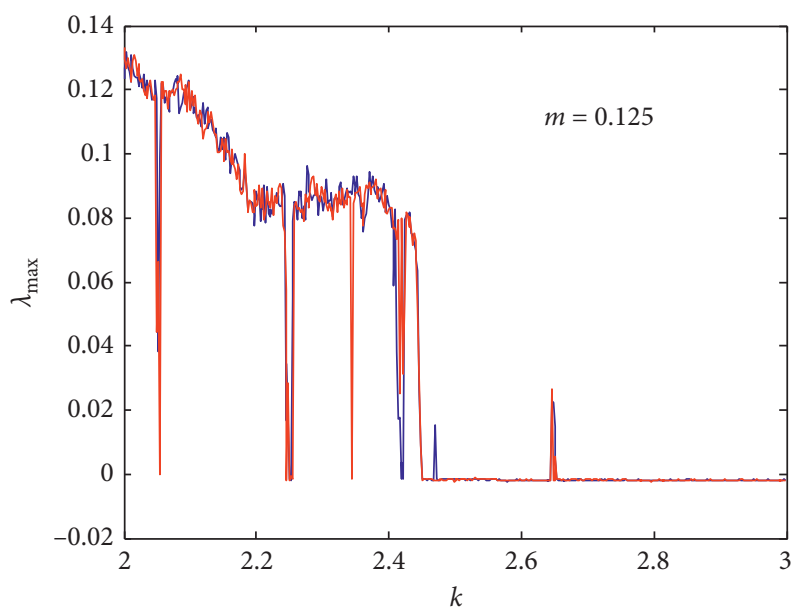

(c)

FIGURE 8: Diagrams of the maximum Lyapunov exponents corresponding to the bifurcation diagrams of Figure 7 showing the impact of the value of $m$ on the symmetry of system (2) considering $k$ as a control parameter.

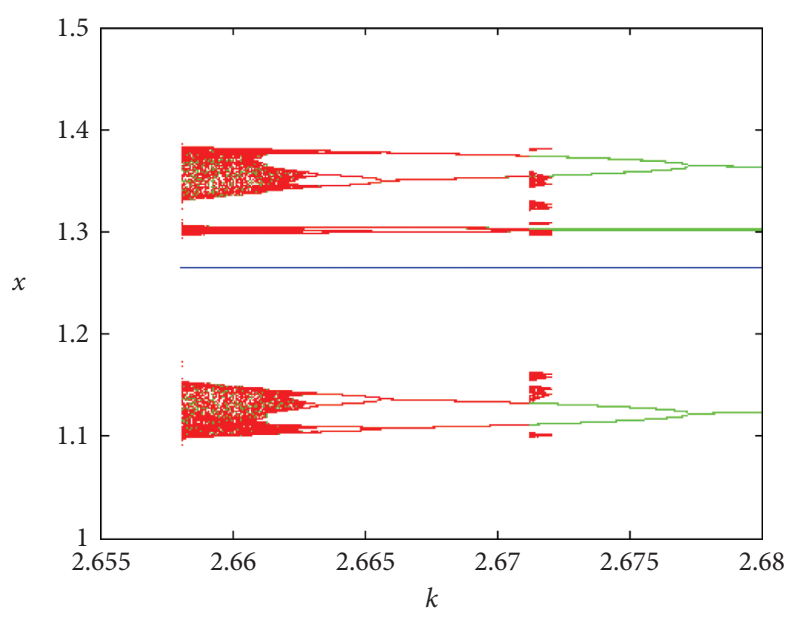

(a)

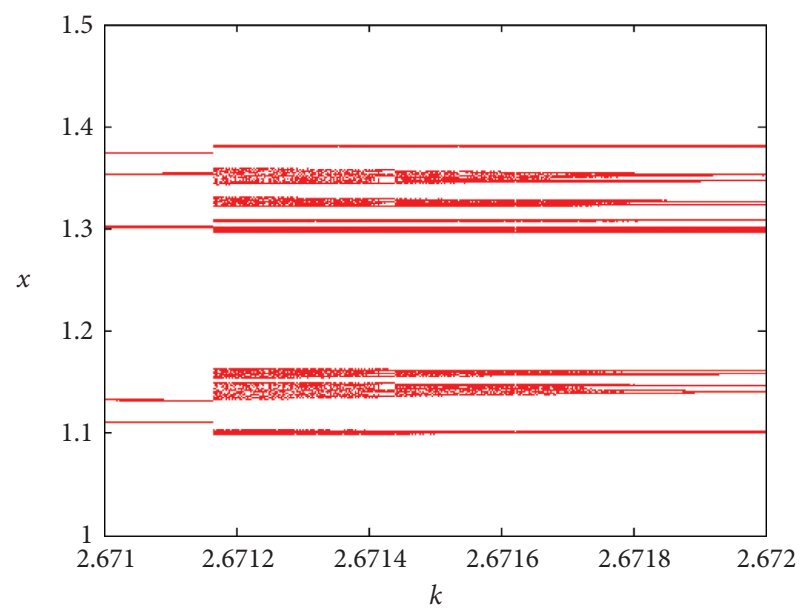

(b)

FiguRE 9: Enlargement of the bifurcation diagram of Figure 7(a), in the intervals $2.6575 \leq k \leq 2.68$ (a) and $2.671 \leq k \leq 2.672$ (b), revealing the coexistence of attractors in the form of parallel branches. 


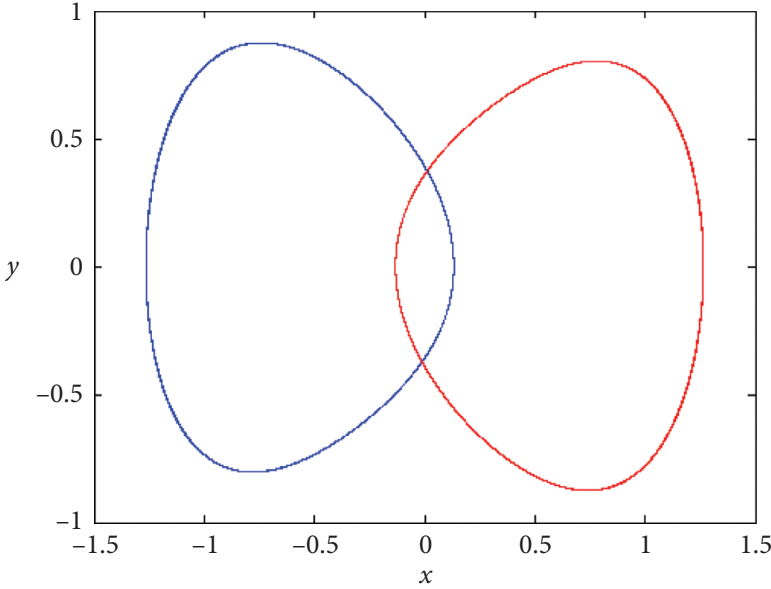

(a)

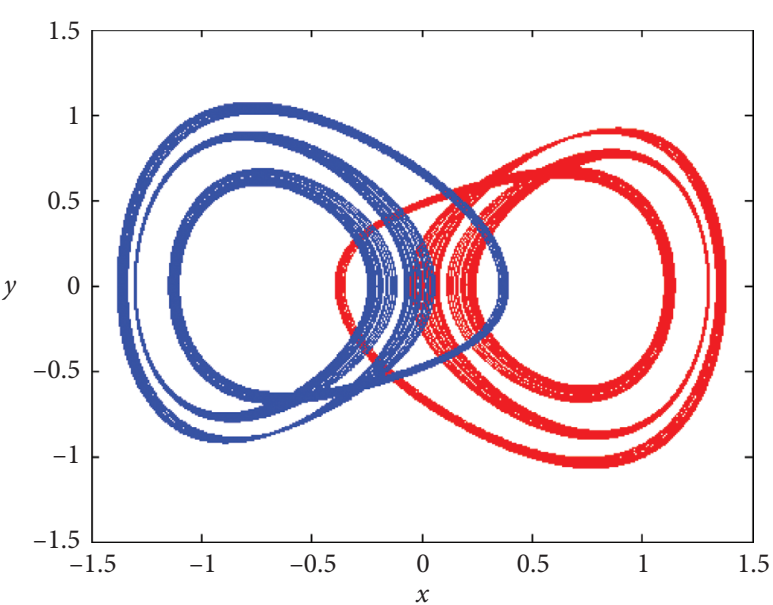

(b)

Figure 10: Coexistence of four attractors at the point $k=2.66$ of Figure 9(a), for different values of the initial conditions $(x(0), y(0), z(0))$ : (a) a period- 1 attractor and its symmetry for $( \pm 0.12,0,0)$; (b) a chaotic spiral attractor and its symmetry for $( \pm 0.39,0,0)$.

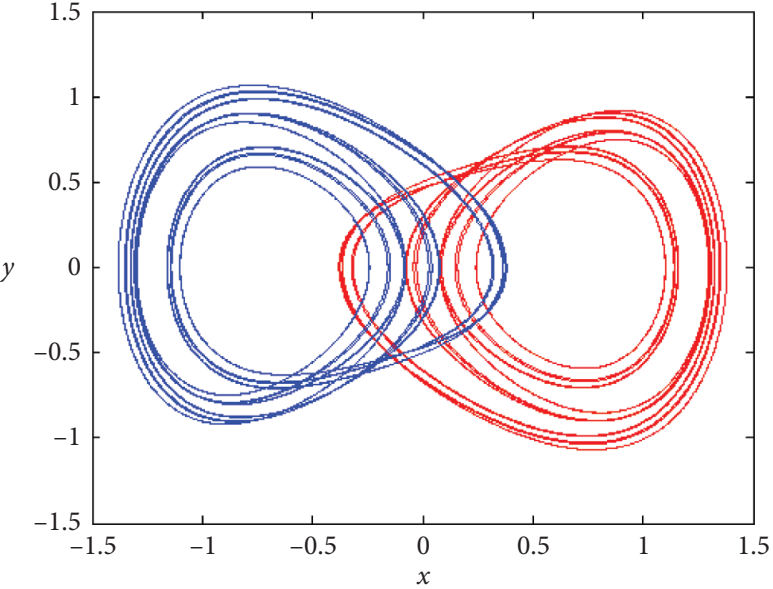

(a)

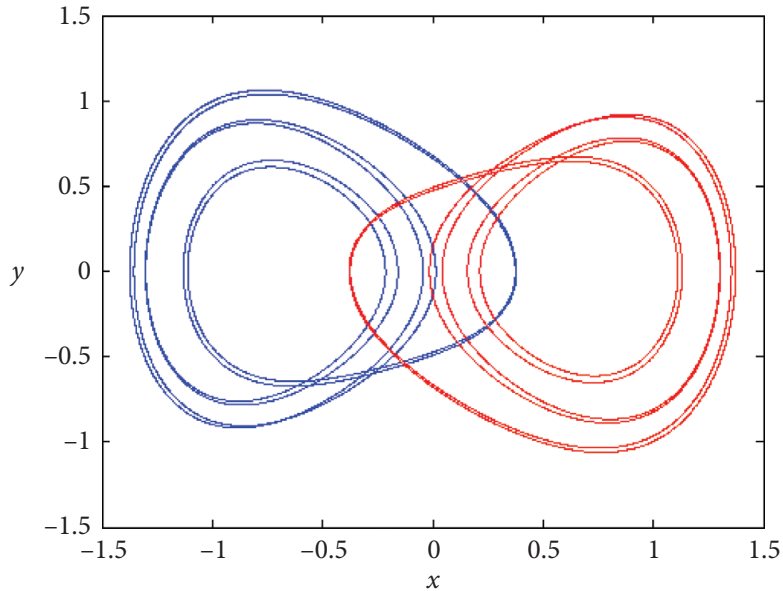

(b)

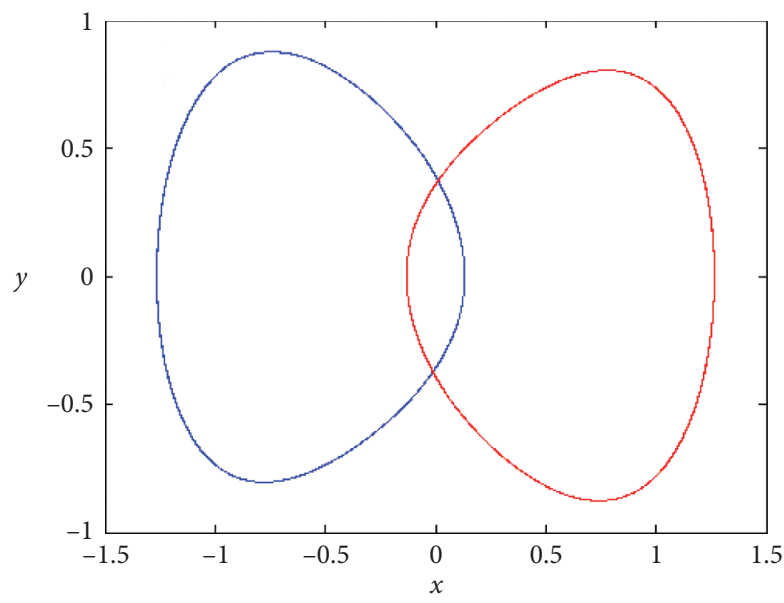

(c)

Figure 11: Coexistence of six periodic attractors at point $k=2.6714$ of Figure 9(b), for different values of the initial conditions $(x(0), y(0)$, $z(0))$ : (a) a finite n-period attractor and its symmetry for $( \pm 0.028,0,0)$; (b) a period-6 attractor and its symmetry for $( \pm 0.06,0,0) ;(c)$ a period- 1 attractor and its symmetry for $( \pm 0.108,0,0)$. 


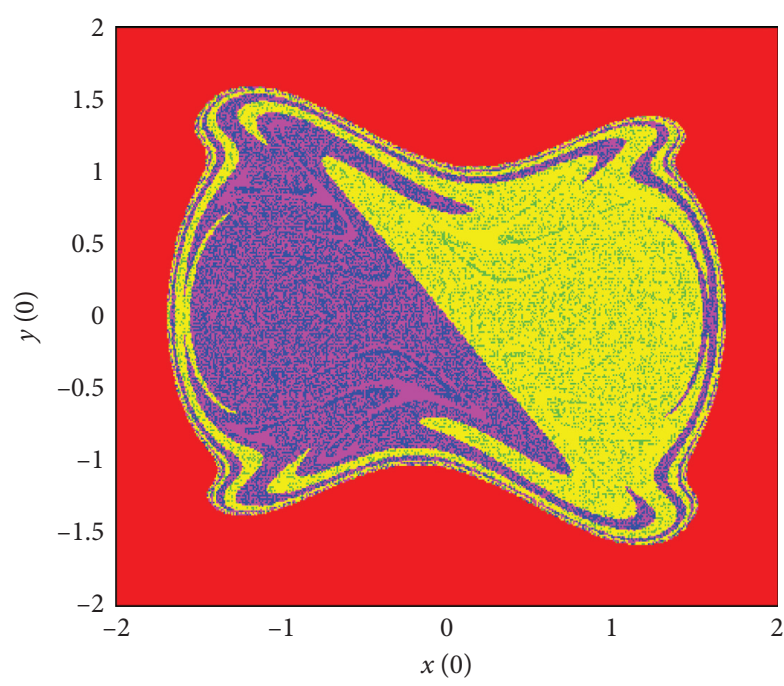

(a)

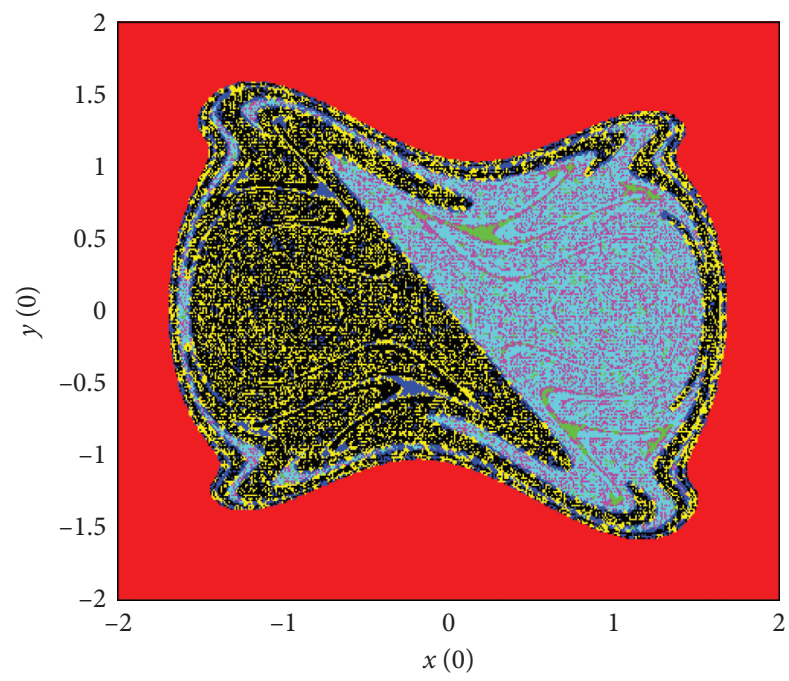

(c)

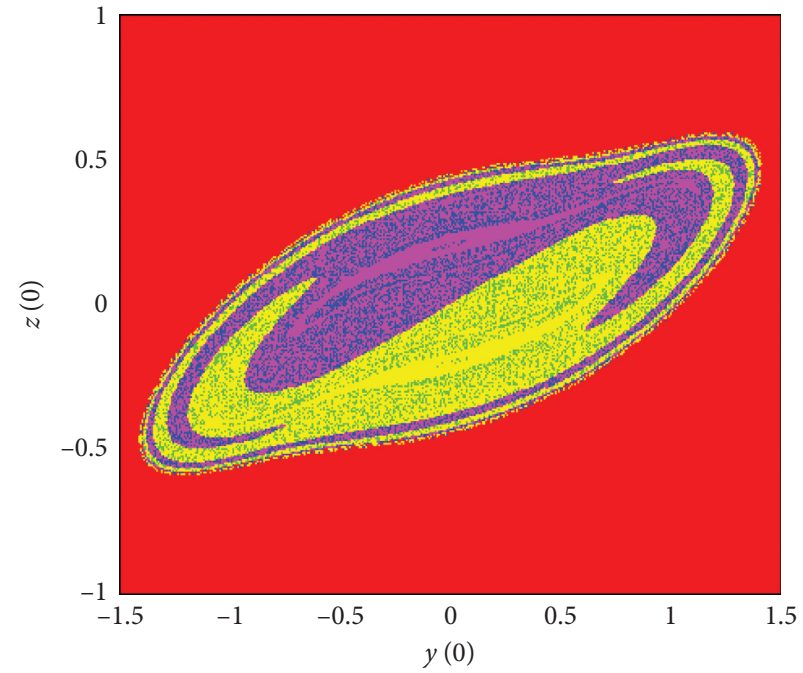

(b)

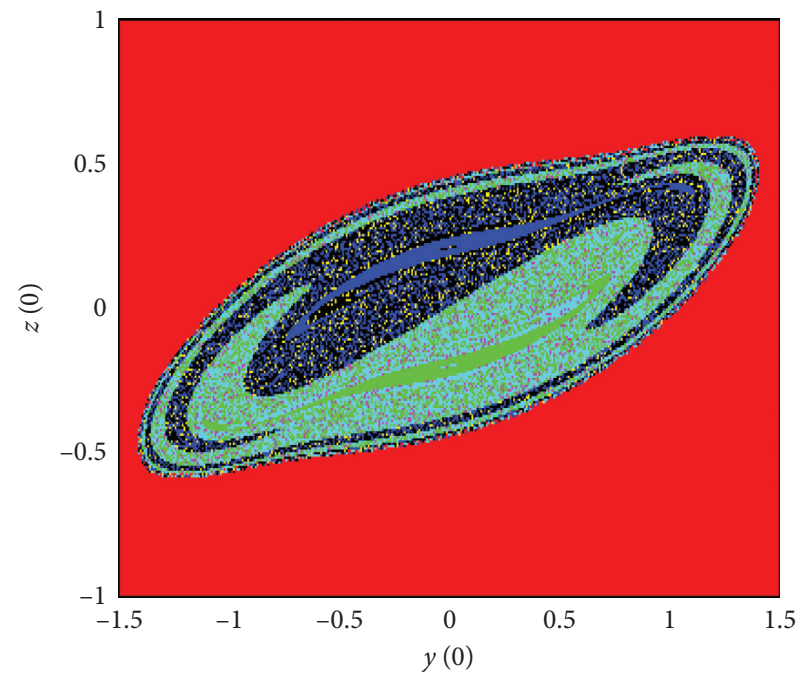

(d)

Figure 12: Cross sections of the basins of attraction in the $(x(0)-y(0))$ and $(y(0)-z(0))$ plane: on $(\mathrm{a}, \mathrm{b})$ blue and yellow colors indicate regions of period-1 while green and magenta indicate regions of chaotic attractors; on (c, d) magenta and yellow colors indicate the regions of n-finite periods, cyan and black indicate the regions of period-6 while green and blue the regions indicate of period-1. Regions of unbounded behavior are in red.

$$
\left\{\begin{array}{l}
\dot{e}_{1}=\dot{x}_{2}-\dot{x}_{1}=e_{2}+u_{1}, \\
\dot{e}_{2}=\dot{y}_{2}-\dot{y}_{1}=e_{1}-\left(x_{2}^{3}-x_{1}^{3}\right)-m\left(x_{2}^{2}-x_{1}^{2}\right)+b e_{2}-k e_{3}+u_{2}, \\
\dot{e}_{3}=\dot{z}_{2}-\dot{z}_{1}=w_{f}\left(e_{2}-e_{3}\right)+u_{3},
\end{array}\right.
$$

where

$$
\begin{aligned}
& e_{2}=y_{2}-y_{1}, \\
& e_{3}=z_{2}-z_{1} .
\end{aligned}
$$

For reasons of simplicity, consider the expressions of the controllers described by

$$
\left\{\begin{array}{l}
u_{1}=-e_{2}-k_{1} e_{1} \\
u_{2}=-e_{1}+\left(x_{2}^{3}-x_{1}^{3}\right)+m\left(x_{2}^{2}-x_{1}^{2}\right)-\widehat{b} e_{2}+\widehat{k} e_{3}-k_{2} e_{2}, \\
u_{3}=-\widehat{w}_{f}\left(e_{2}-e_{3}\right)-k_{3} e_{3}
\end{array}\right.
$$

with $\widehat{b}, \widehat{w}_{f}$, and $\widehat{k}$ being the estimated parameters of $b, w_{f}$, and $k$, respectively. $k_{i}(i=1,2,3)$ is the positive feedback gain.

By substituting (22) in (19) and (20), we obtain the following expressions for the slave subsystem (23) and the synchronization errors (24): 


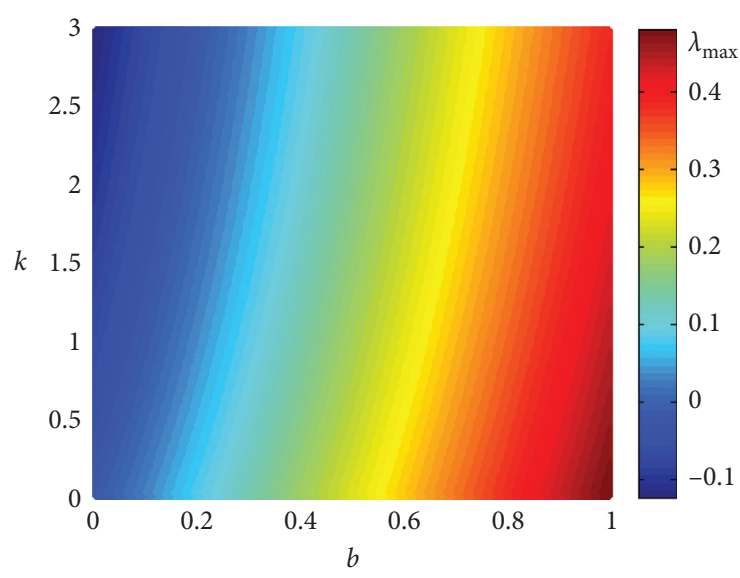

(a)

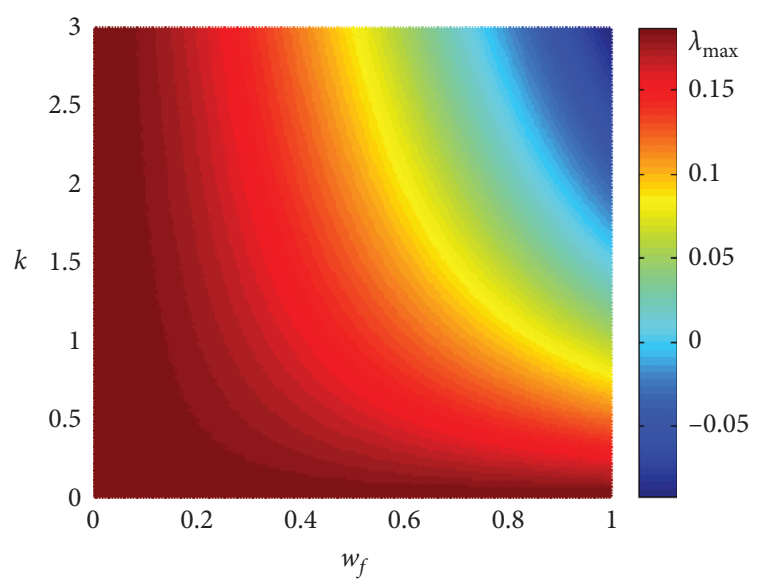

(b)

FigURE 13: Behaviors of system (2) with respect to $(b-k),\left(w_{f}-k\right)$ and maximum Lyapunov exponents $\left(\lambda_{\max }\right)$, for $m=0$ : the periodic zones (where $\left.\lambda_{\max }<0\right)$ are in blue and the other zones (where $\lambda_{\max }>0$ ) are chaotic.

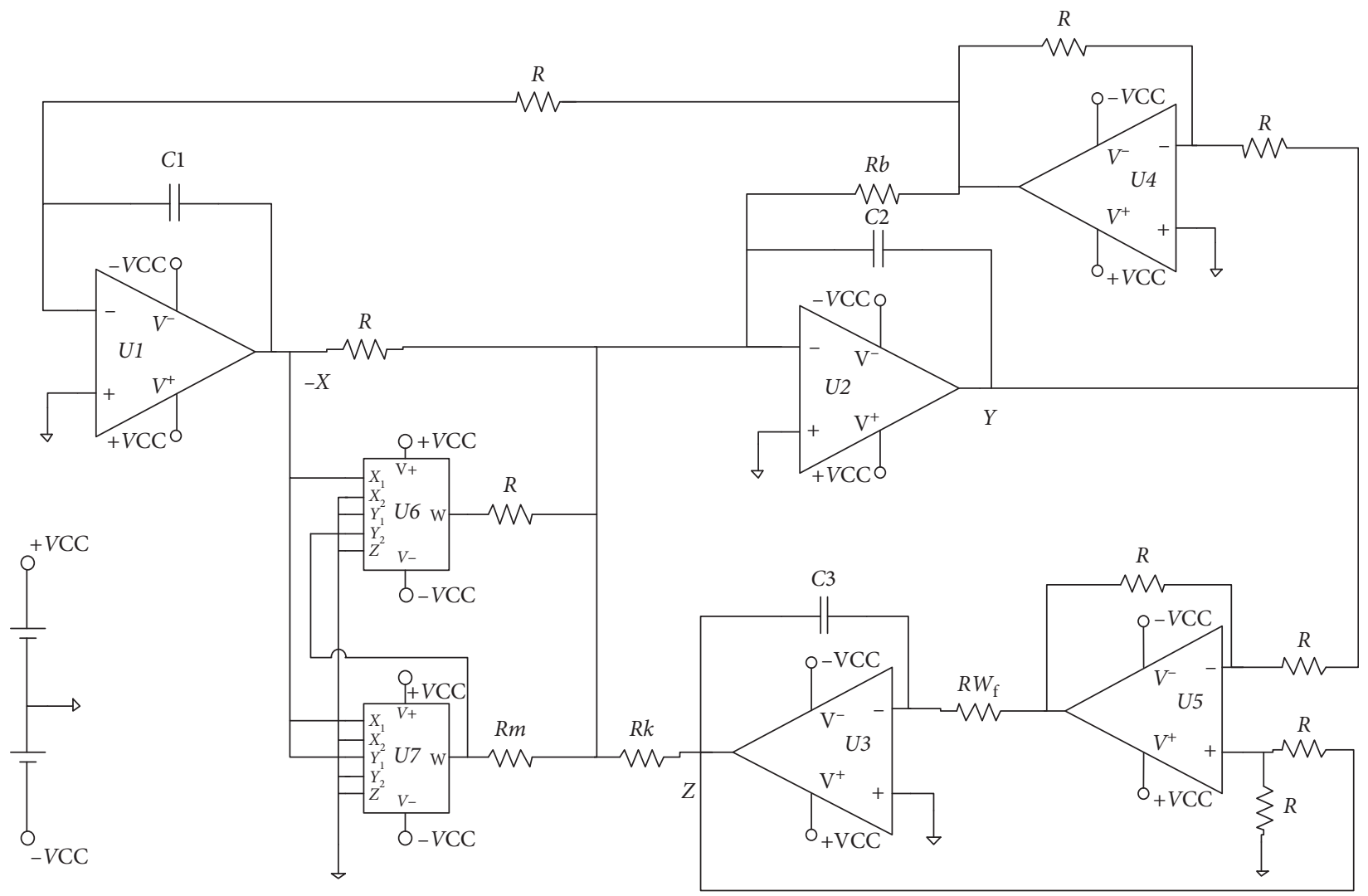

Figure 14: Circuit realization of the three-dimensional autonomous Duffing-Holmes like oscillator described by system $(2)$ where $U_{i}(i=$ $1,2,3,4,5)$ are TL084CN and $U_{j}(j=6,7)$ are AD633JN. 
TABLE 3: Comparison between the results of numerical integrations and PSpice.

\begin{tabular}{|c|c|c|c|c|c|c|c|}
\hline \multirow{2}{*}{ System } & \multirow{2}{*}{ Attractors } & \multicolumn{4}{|c|}{ Numerical integration results } & \multicolumn{2}{|c|}{ PSpice results } \\
\hline & & $b$ & $R_{b}=10 / b(k \Omega)$ & $k$ & $R_{k}=10 / k(\mathrm{k} \Omega)$ & $R_{b}(\mathrm{k} \Omega)$ & $R_{k}(\mathrm{k} \Omega)$ \\
\hline \multirow{7}{*}{ Symmetric $\left(m=0.0, R_{m}=1 \mathrm{M} \Omega\right)$} & Period 1 & 0.22 & 45.4545 & \multirow{5}{*}{1.6} & \multirow{5}{*}{6.25} & 45 & \multirow[t]{5}{*}{6.25} \\
\hline & Period 2 & 0.28 & 35.7142 & & & 35.6 & \\
\hline & Period 4 & 0.284 & 35.2112 & & & 35.4 & \\
\hline & Single band chaos & 0.288 & 34.7222 & & & 35 & \\
\hline & Double band chaos & 0.3 & 33.3333 & & & 33.33 & \\
\hline & Coexistence of four & \multirow{2}{*}{0.41} & \multirow{2}{*}{24.3902} & \multirow{2}{*}{$\begin{array}{c}2.66 \\
2.6714 \\
\end{array}$} & \multirow{2}{*}{$\begin{array}{l}3.7593 \\
3.7433 \\
\end{array}$} & 24.39 & 3.746 \\
\hline & Coexistence of six & & & & & 24.39 & 3.745 \\
\hline \multirow{6}{*}{ Asymmetric $\left(m=0.05, R_{m}=200 \mathrm{k} \Omega\right)$} & Period 1 & 0.22 & 45.4545 & \multirow{6}{*}{1.6} & \multirow{6}{*}{6.25} & 37 & \multirow{6}{*}{6.25} \\
\hline & Period 2 & 0.25 & 40 & & & 36 & \\
\hline & Period 4-period 2 & 0.2824 & 35.4107 & & & 35.6 & \\
\hline & Single band chaos-period 2 & 0.2856 & 35.01 & & & 35.2 & \\
\hline & Single band chaos & 0.2892 & 34.5781 & & & 34.8 & \\
\hline & Double band chaos & 0.3 & 33.3333 & & & 34.631 & \\
\hline
\end{tabular}

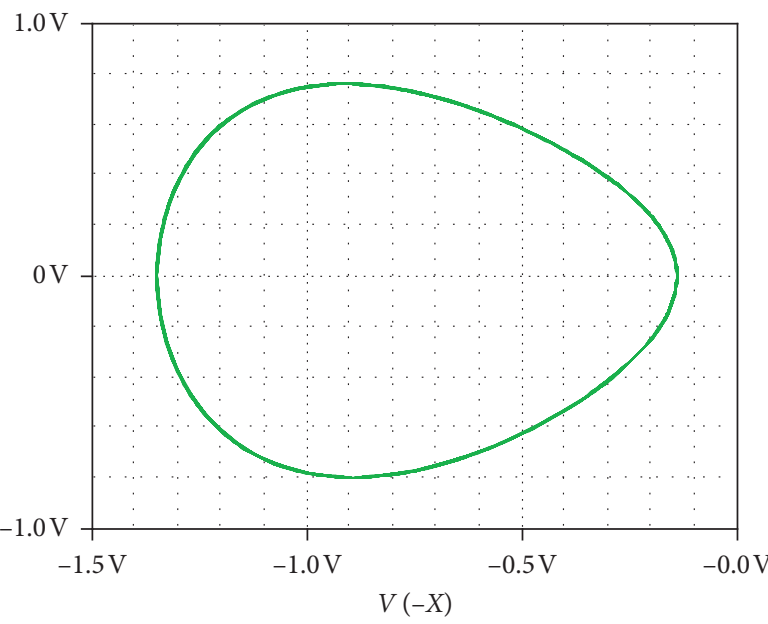

$V(Y)$

(a)

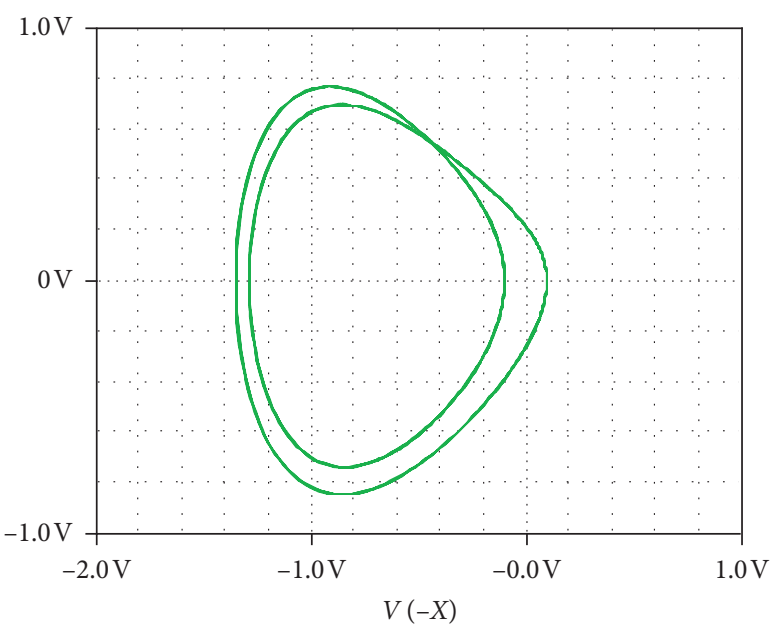

$\checkmark V(Y)$

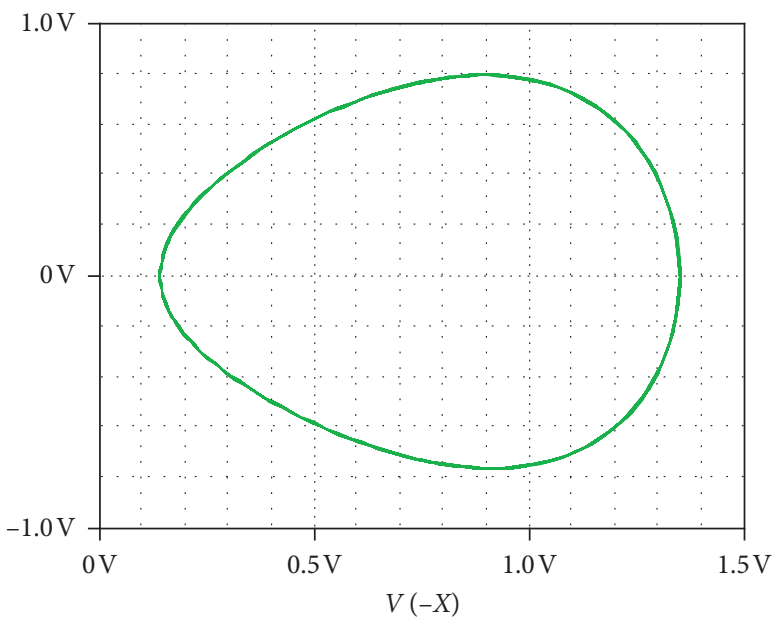

$V(Y)$

(b)

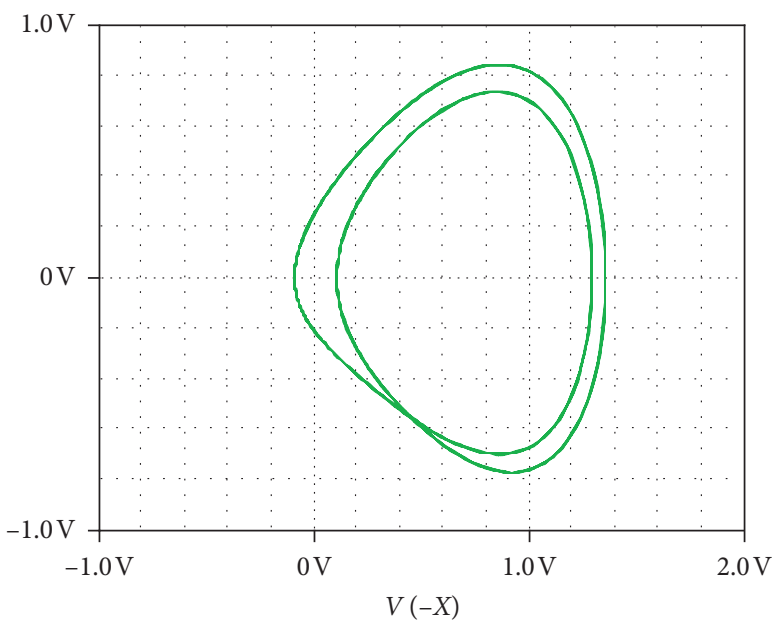

$V(Y)$

(c) 


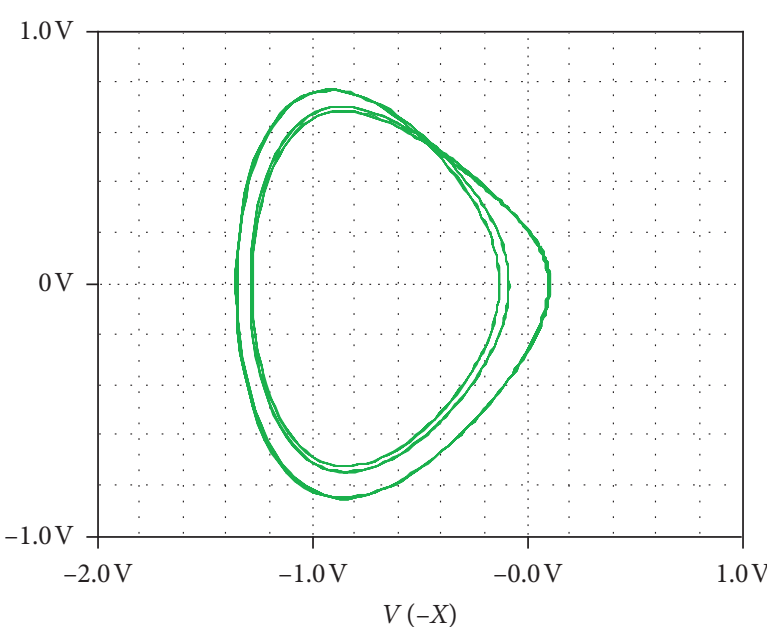

$\square V(Y)$

(e)

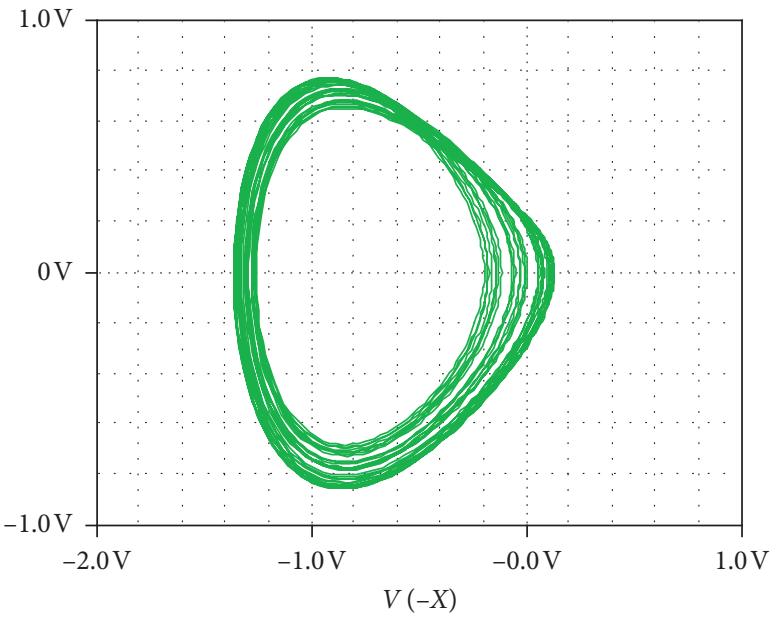

$V(Y)$

(g)

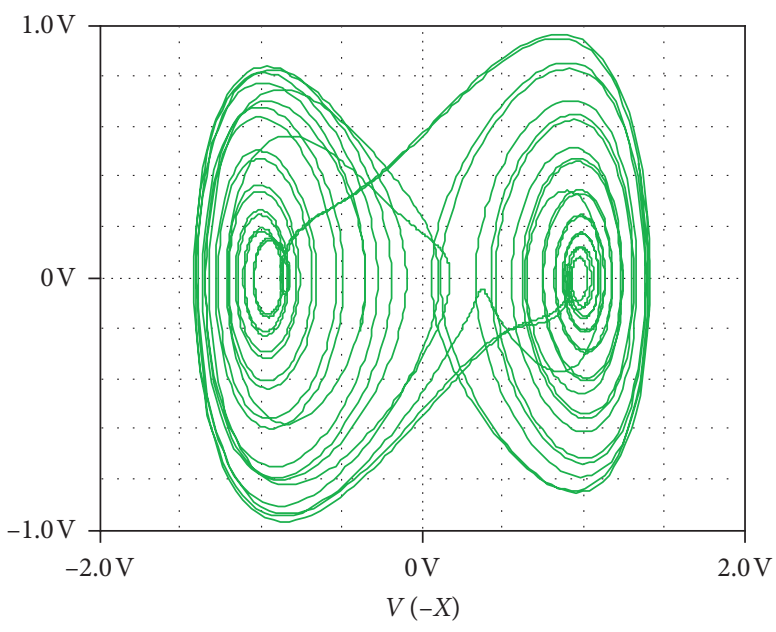

$V(Y)$

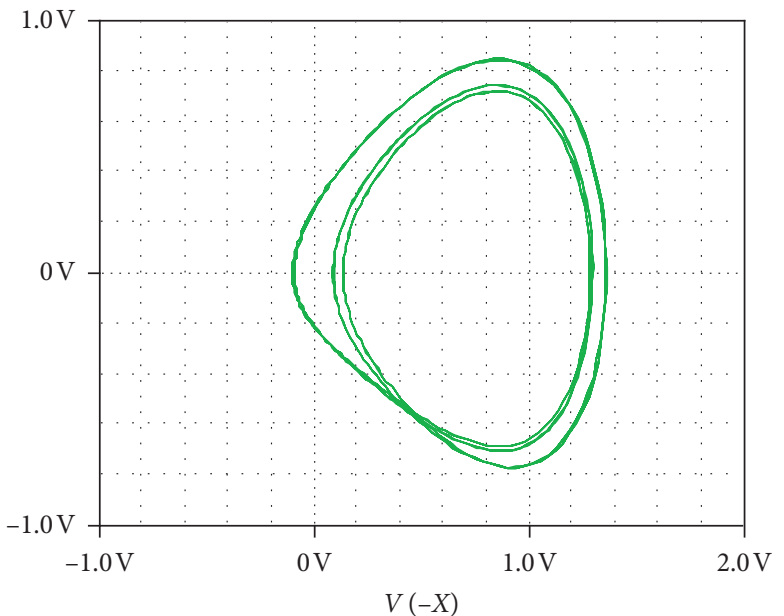

$\square V(Y)$

(f)

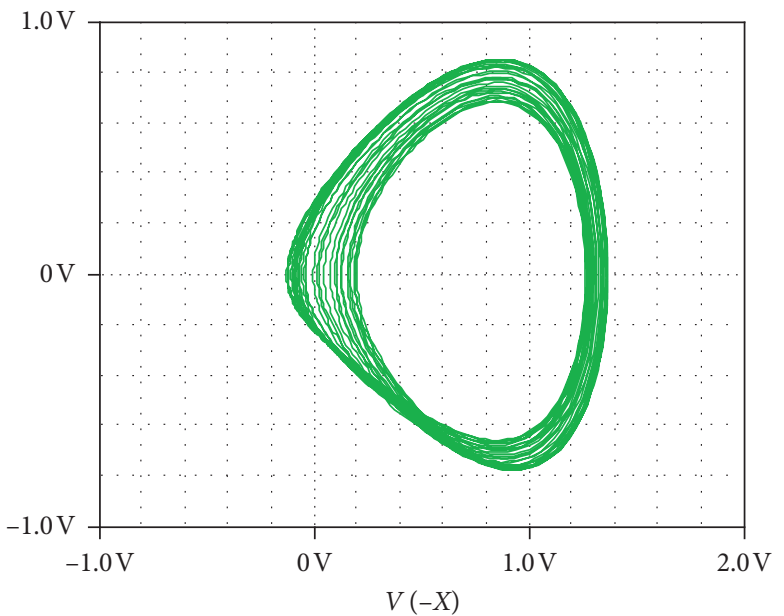

$V(Y)$

(h)

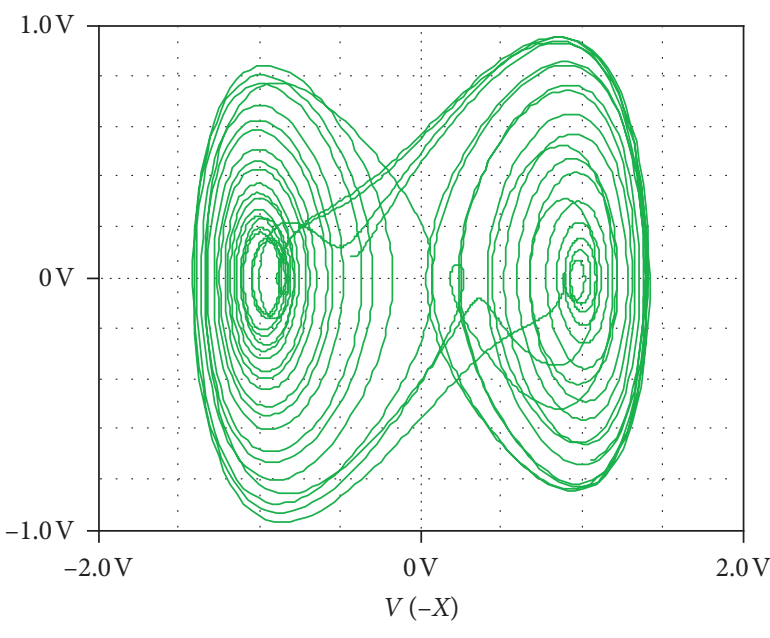

$V(Y)$

(i)

(j)

FiguRE 15: Phase portraits obtained from PSpice for $R_{m}=1 \mathrm{M} \Omega$ (corresponding to the symmetric case) and different values of the control resistance $R_{b}$ showing (a) cycle of period- 1 for $R_{b}=45 \mathrm{k} \Omega$, (b) cycle of period- 2 for $R_{b}=35.6 \mathrm{k} \Omega$, (c) cycle of period- 4 for $R_{b}=35.4 \mathrm{k} \Omega$, (d) single chaotic band for $R_{b}=35 \mathrm{k} \Omega$, and (e) double-band chaotic attractor $R_{b}=33.33 \mathrm{k} \Omega$. The pairs are obtained for $(X(0), Y(0), Z(0))=( \pm 0.25,0,0)$. 


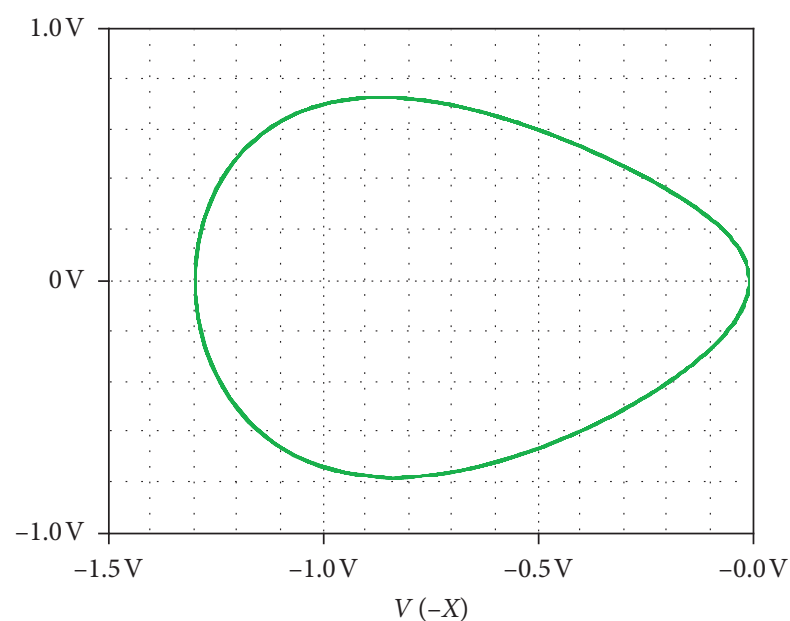

$\square V(Y)$

(a)

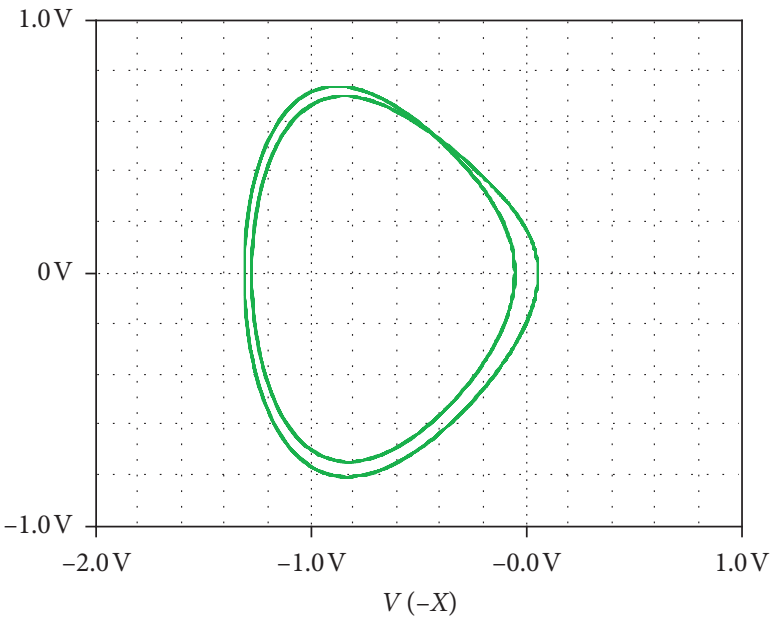

$\square V(Y)$

(c)

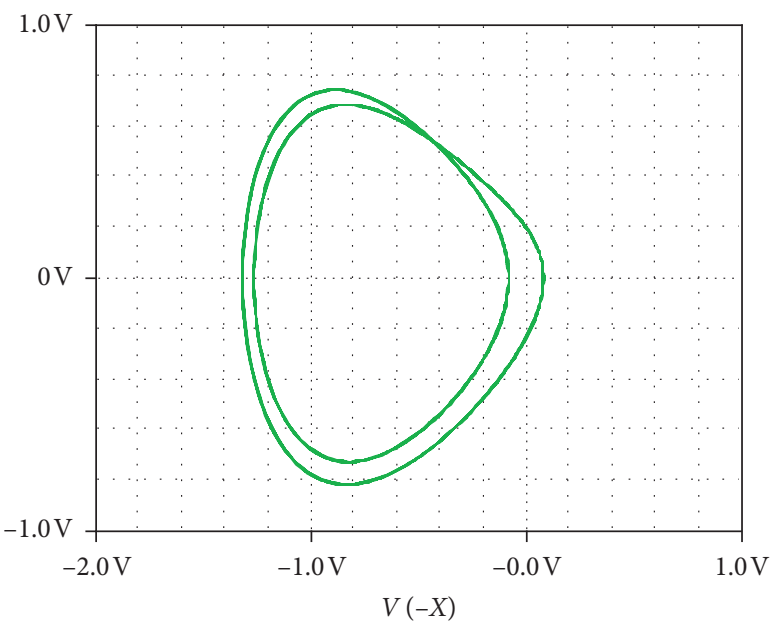

$\square V(Y)$

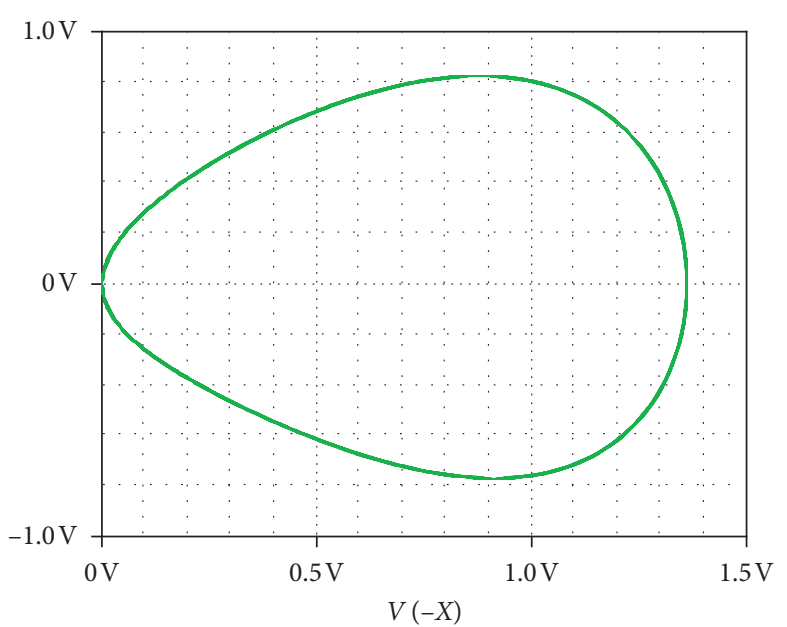

$\square V(Y)$

(b)

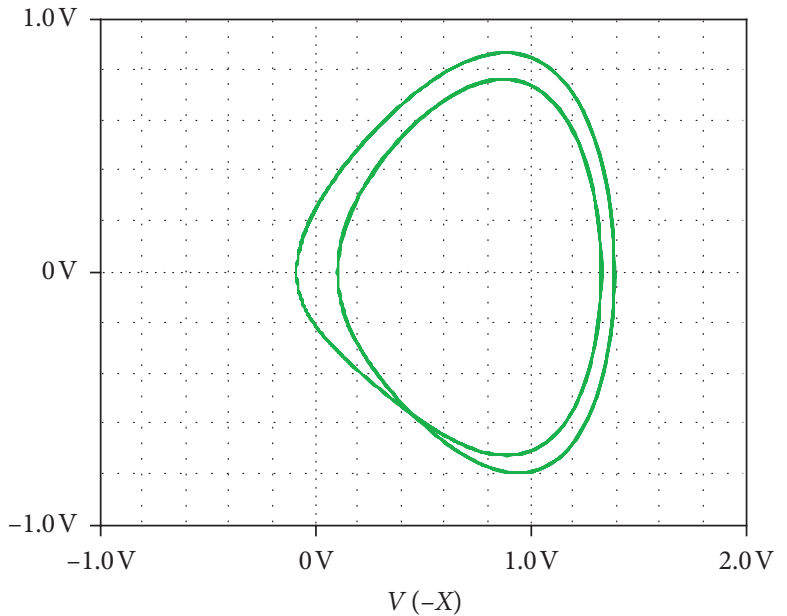

$\square V(Y)$

(d)

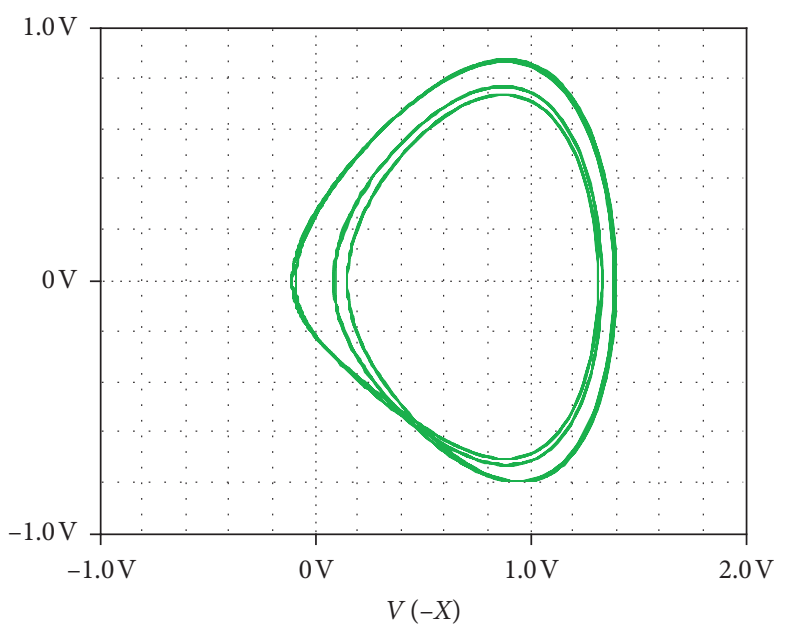

$\square V(Y)$

(e)

FIgURE 16: Continued. 


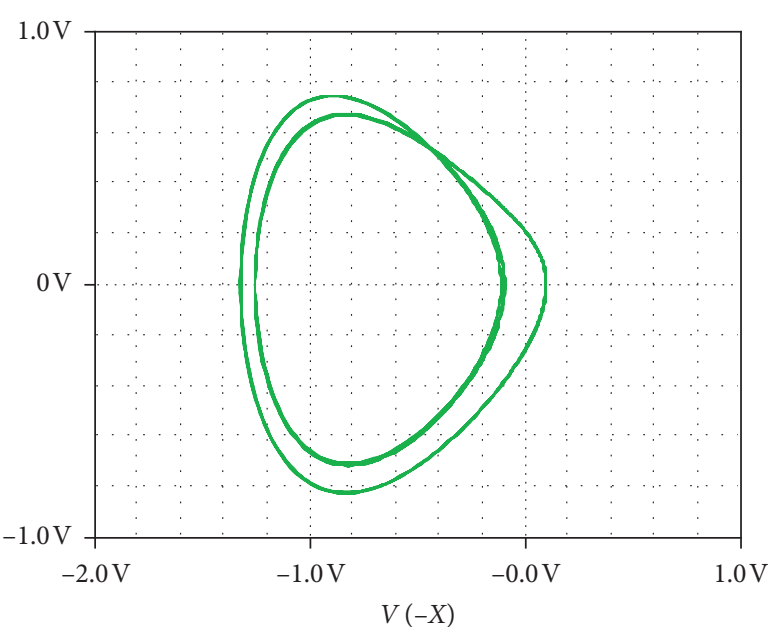

$\square V(Y)$

(g)

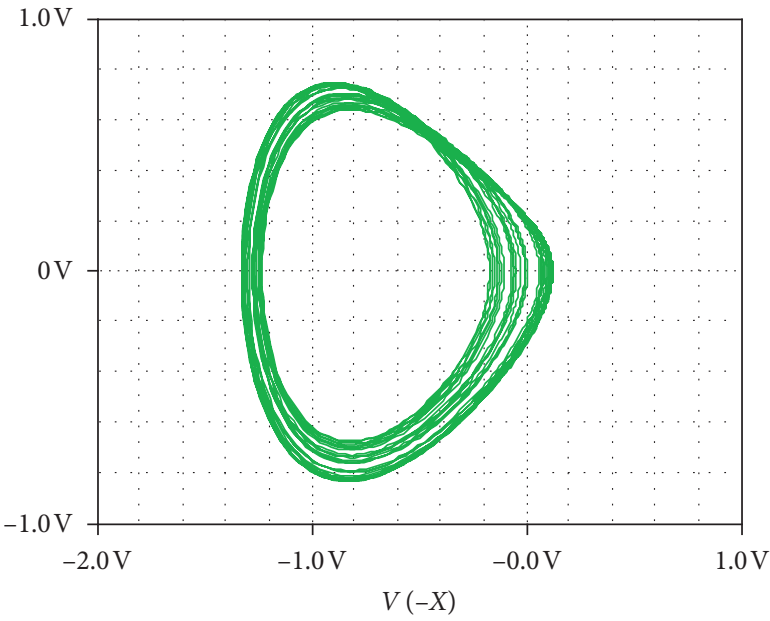

$V(Y)$

(i)

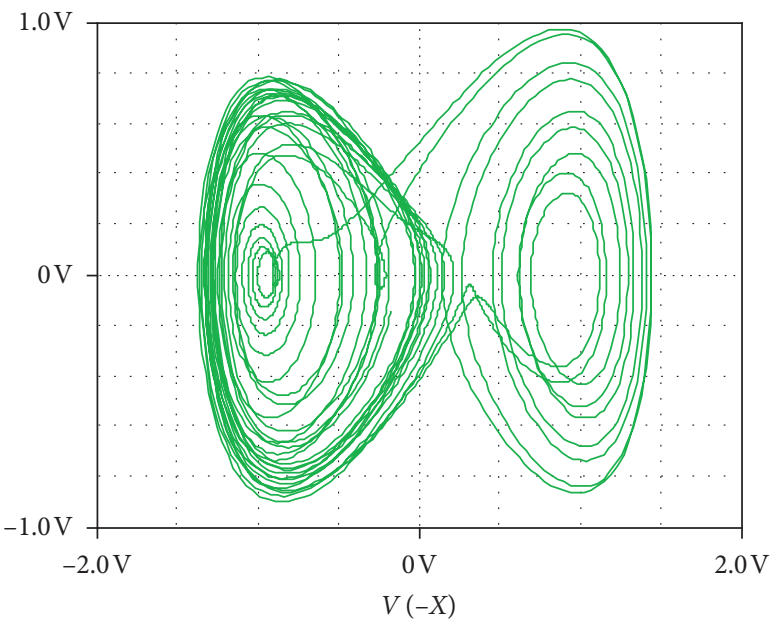

$V(Y)$

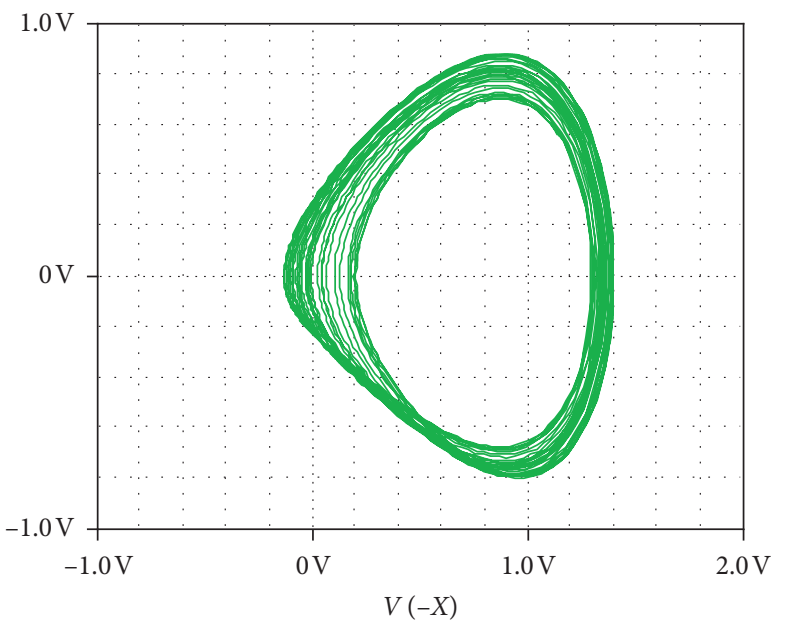

$V(Y)$

(h)

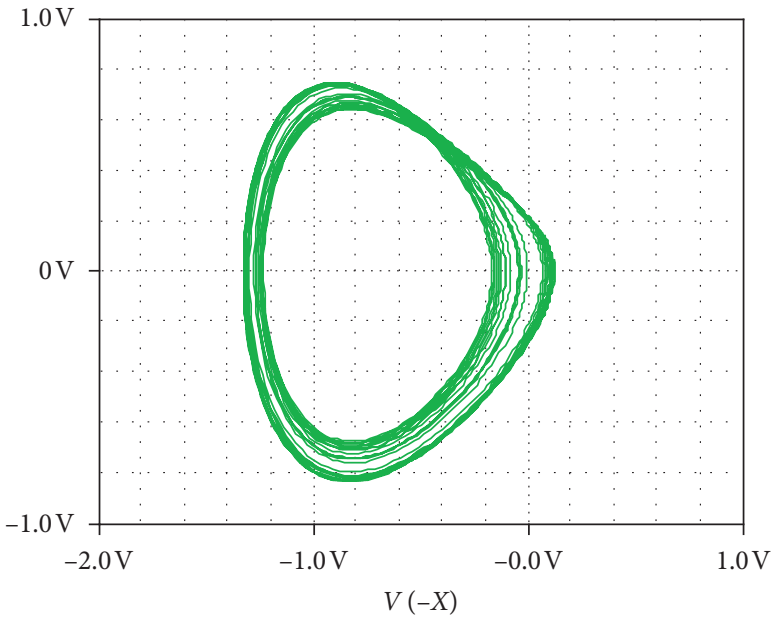

$V(Y)$

(j)

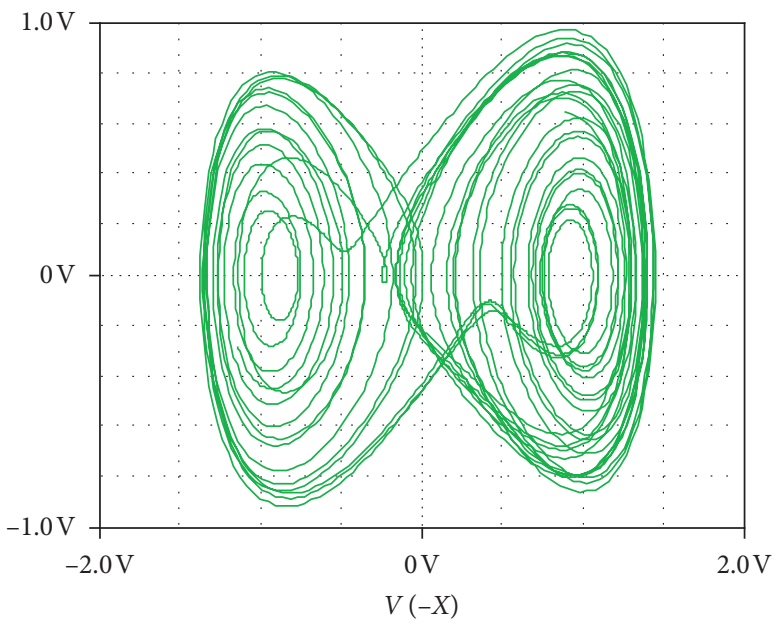

$V(Y)$

(k)

(1)

Figure 16: Phase portraits obtained from PSpice for $R_{m}=200 \mathrm{k} \Omega$ (corresponding to the asymmetric case) and different values of the control resistance $R_{b}$ showing (a,b) cycle of period- 1 for $R_{b}=37 \mathrm{k} \Omega$, (c, d) cycle of period- 2 for $R_{b}=36 \mathrm{k} \Omega$; (e) and (f) for cycle of period- 4 and period-2 respectively with $R_{b}=35.6 \mathrm{k} \Omega ;(\mathrm{g})$ and $(\mathrm{h})$ for single-band chaotic attractor and cycle of period-4 respectively with $R_{b}=35.2 \mathrm{k} \Omega$; $(\mathrm{i}$, j) for single-band chaotic attractor with $R_{b}=34.8 \mathrm{k} \Omega$; (k) and (l) for two asymmetric double-band chaotic attractor with $R_{b}=34.631 \mathrm{k} \Omega$. The pairs are obtained for $(X(0), Y(0), Z(0))=( \pm 0.15,0,0)$. 


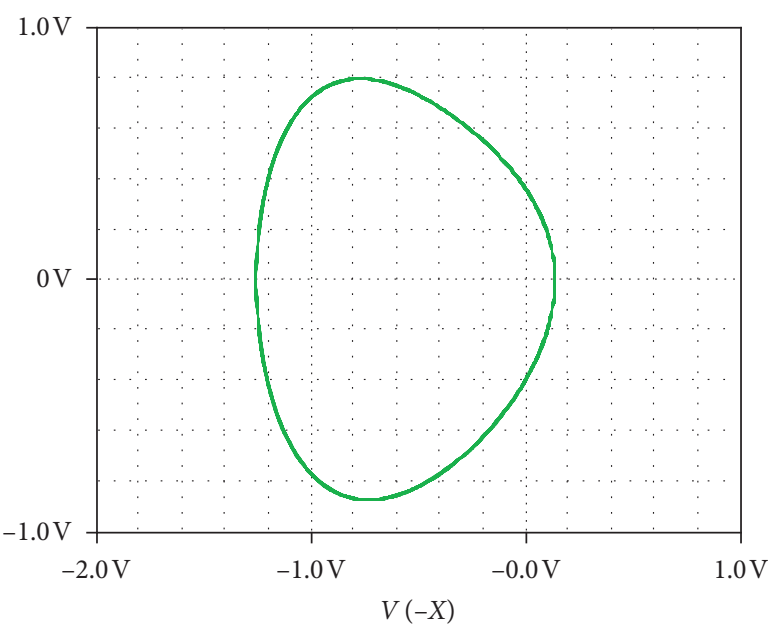

$\square(Y)$

(a)

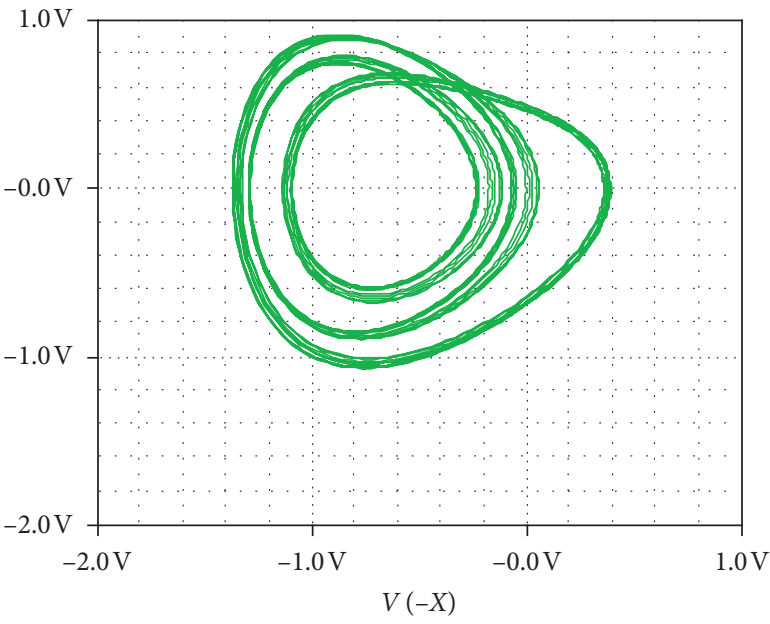

$V(Y)$

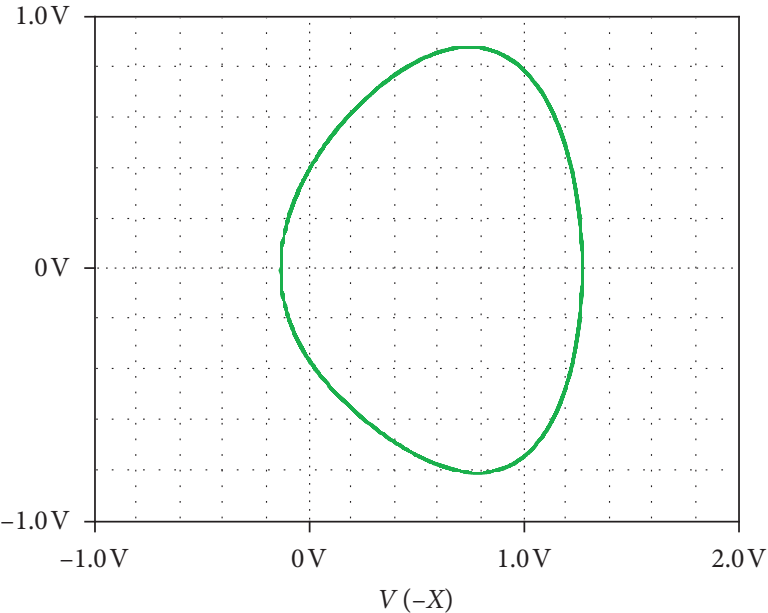

$\square V(Y)$

(b)

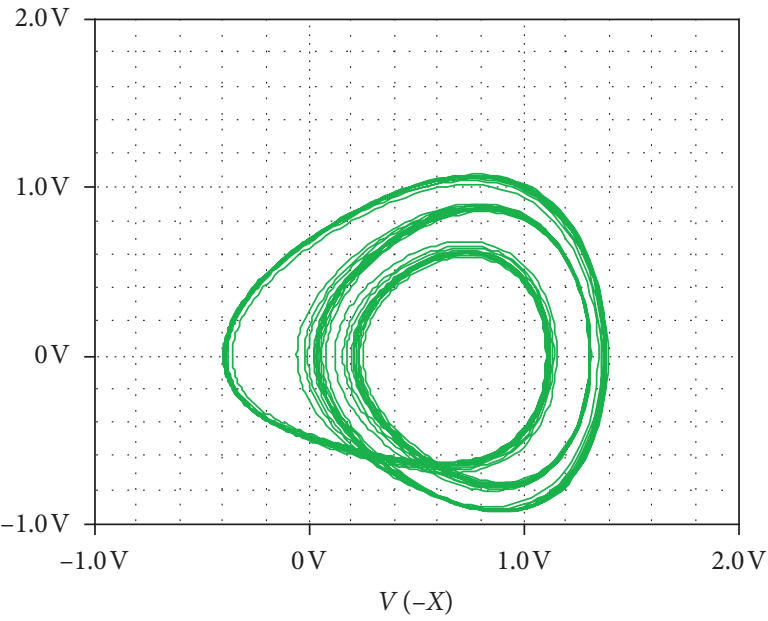

$V(Y)$

(c)

(d)

FIGURE 17: Representations of the four coexisting attractors obtained in PSpice with $R_{m}=1 \mathrm{M} \Omega, R_{k}=3.746 \mathrm{k} \Omega$ for different values of the initial conditions $(X(0), Y(0), Z(0))$ : (a) cycle of period-1 and (b) its symmetry for $( \pm 0.13,0,0)$; (c) chaotic attractor and (d) its symmetry for $( \pm 0.15,0,0)$.

$$
\begin{aligned}
& \left\{\begin{array}{l}
\dot{x}_{2}=y_{1}-k_{1} e_{1} \\
\dot{y}_{2}=x_{1}-x_{1}^{3}-m x_{1}^{2}+y_{2} e_{b}+\widehat{b} y_{1}-z_{2} e_{k}-\widehat{k} z_{1}-k_{2} e_{2} \\
\dot{z}_{2}=\left(y_{2}-z_{2}\right) e_{w_{f}}+\widehat{w}_{f}\left(y_{1}-z_{1}\right)-k_{3} e_{3}
\end{array}\right. \\
& \left\{\begin{array}{l}
\dot{e}_{1}=-k_{1} e_{2} \\
\dot{e}_{2}=e_{2} e_{b}-e_{3} e_{k}-k_{2} e_{2} \\
\dot{e}_{3}=\left(e_{2}-e_{3}\right) e_{w_{f}}-k_{3} e_{3}
\end{array}\right.
\end{aligned}
$$

where

$$
\left\{\begin{array}{l}
e_{b}=(b-\widehat{b}), \\
e_{w_{f}}=\left(w_{f}-\widehat{w}_{f}\right), \\
e_{k}=(k-\widehat{k}),
\end{array}\right.
$$

such as

$$
\left\{\begin{array}{l}
\dot{e}_{b}=-\dot{\widehat{b}}, \\
\dot{e}_{w_{f}}=-\dot{\widehat{w}}_{f}, \\
\dot{e}_{k}=-\dot{\hat{k}} .
\end{array}\right.
$$

The synchronization problem amounts to impose that the synchronization error $e(\tau) \longrightarrow 0$ (where $e=\left[e_{1}, e_{2}\right.$, $\left.e_{3}\right]^{T}$ ) when $\tau \longrightarrow \infty$ (asymptotically stable around its point of equilibrium) [72]. For this, let us consider the following positive definite function as Lyapunov candidate function:

$$
V\left(e_{1}, e_{2}, e_{3}, e_{b}, e_{w_{f}}, e_{k}\right)=\frac{1}{2}\left(e_{1}^{2}+e_{2}^{2}+e_{3}^{2}+e_{b}^{2}+e_{w_{f}}^{2}+e_{k}^{2}\right) .
$$




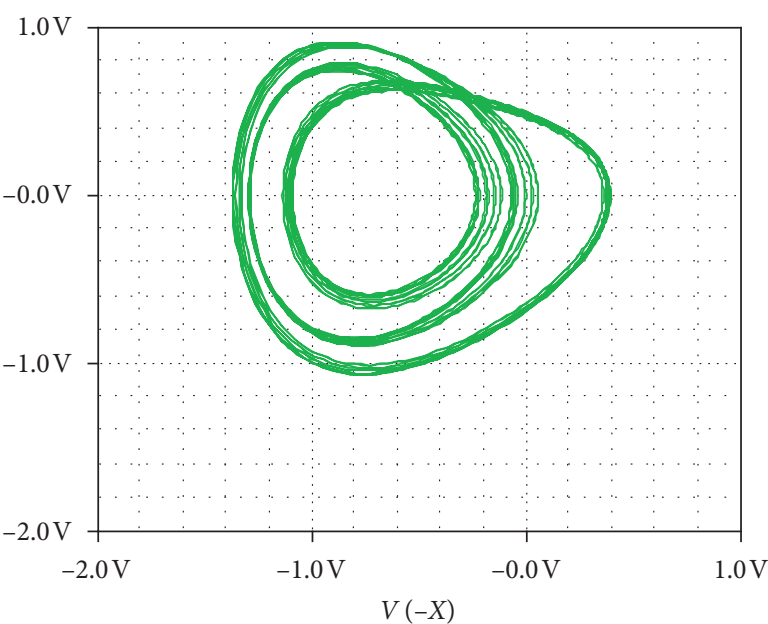

$\square V(Y)$

(a)

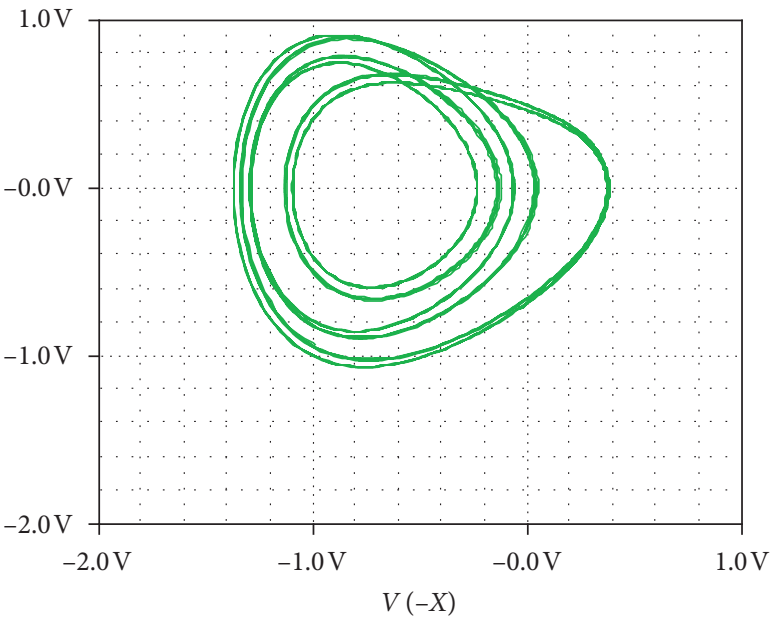

$V(Y)$

(c)

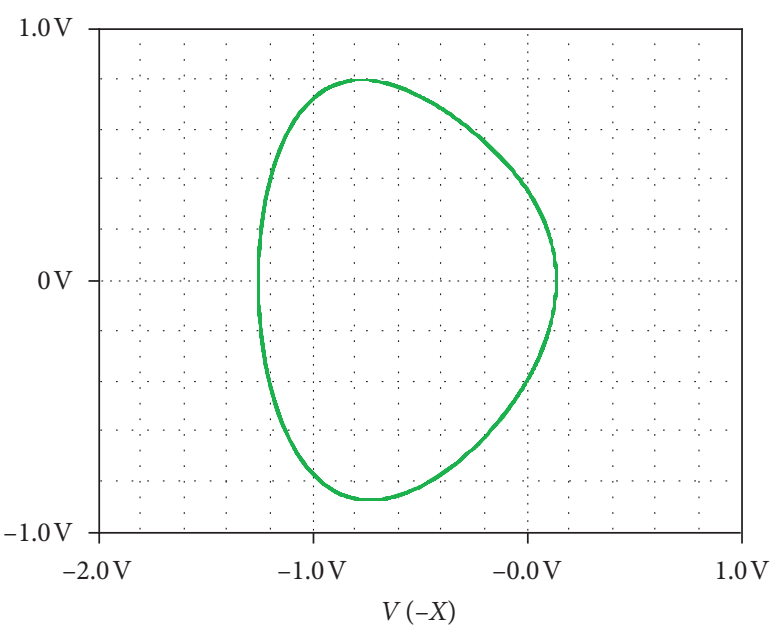

$\square V(Y)$

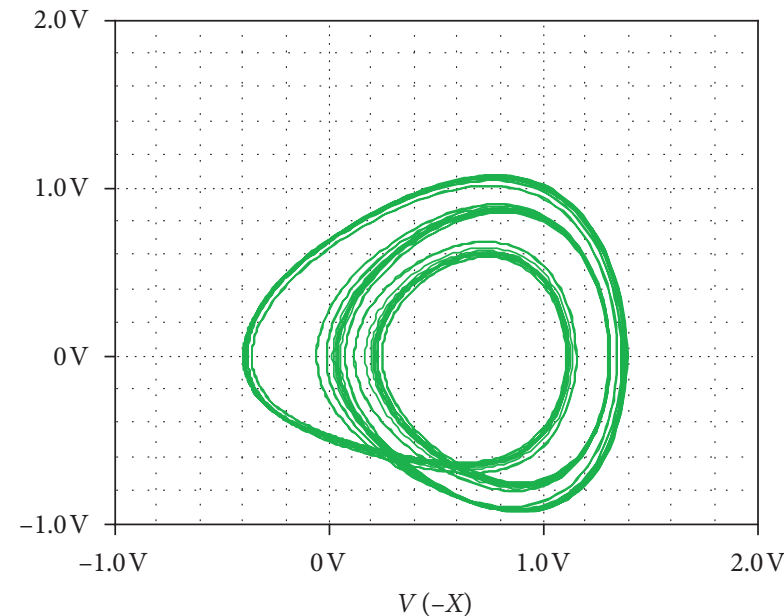

$\square V(Y)$

(b)

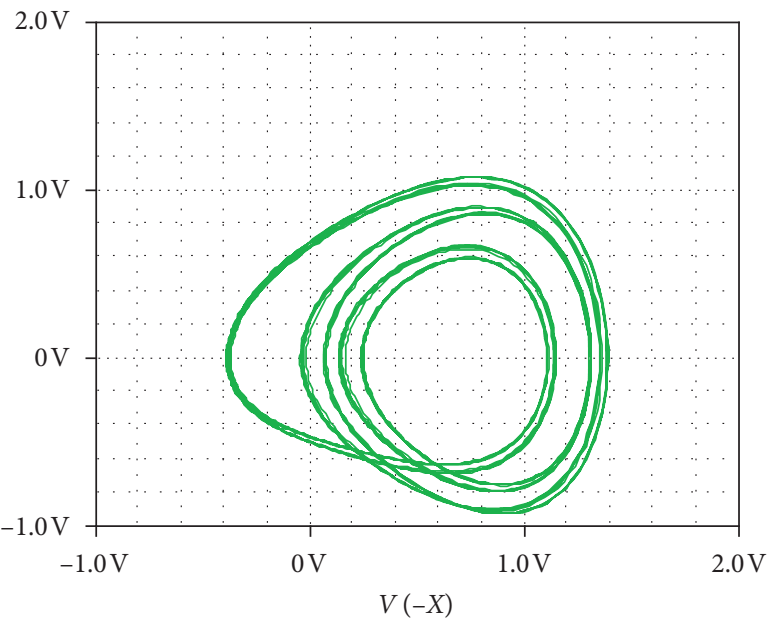

$V(Y)$

(d)

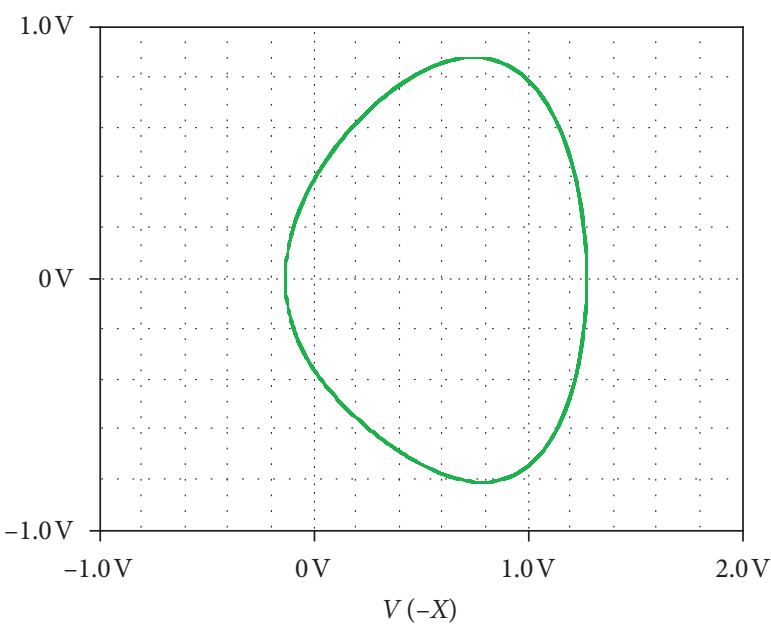

$\square V(Y)$

(e)

(f)

Figure 18: Representation of the six coexistence attractors obtained in PSpice with $R_{m}=1 \mathrm{M} \Omega, R_{k}=3.745 \mathrm{k} \Omega$ for different values of the initial conditions $(X(0), Y(0), Z(0))$ : (a) cycle of $\mathrm{n}$-finite period and (b) its symmetry for $( \pm 0.25,0,0)$; (c) cycle of period-6 and (d) its symmetry for $(+0.17,0,0)$ and $(-0,88,0,0)$, respectively; (e) cycle of period- 1 and (f) its symmetry for $( \pm 0.11,0,0)$. 


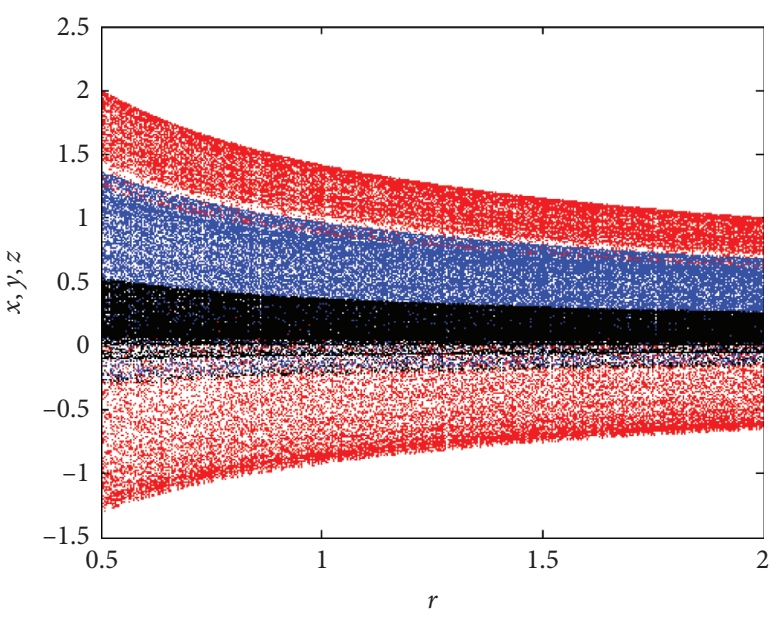

(a)

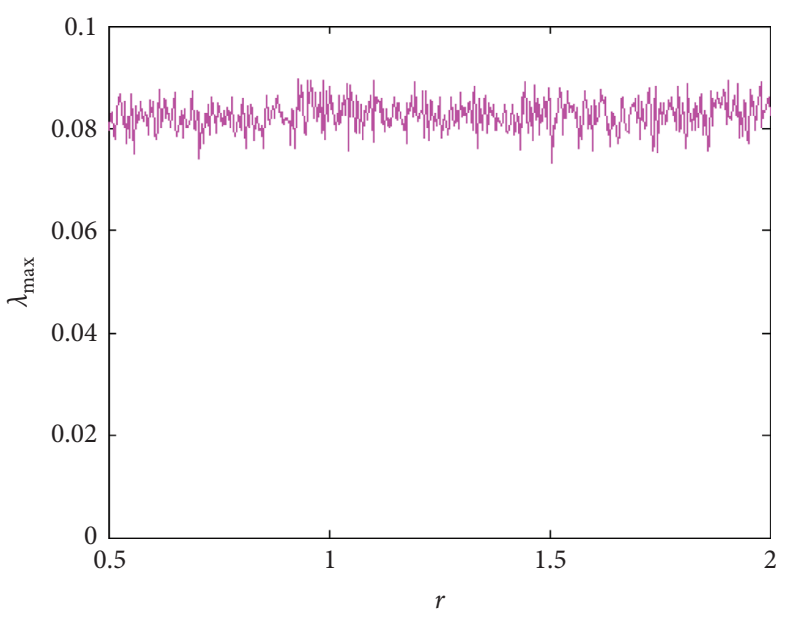

(b)

FIGURe 19: Bifurcation diagrams (a) and largest Lyapunov exponent (b), illustrating the change in amplitude of the variables $x, y$, and $z$ (in red, blue, and black, respectively) as a function of the parameter $r$, without alteration of the properties of the system. The parameters $b, w_{f}, k$, and $m$ are set to $0.3,0.5,1.6$, and 0.0 , respectively.

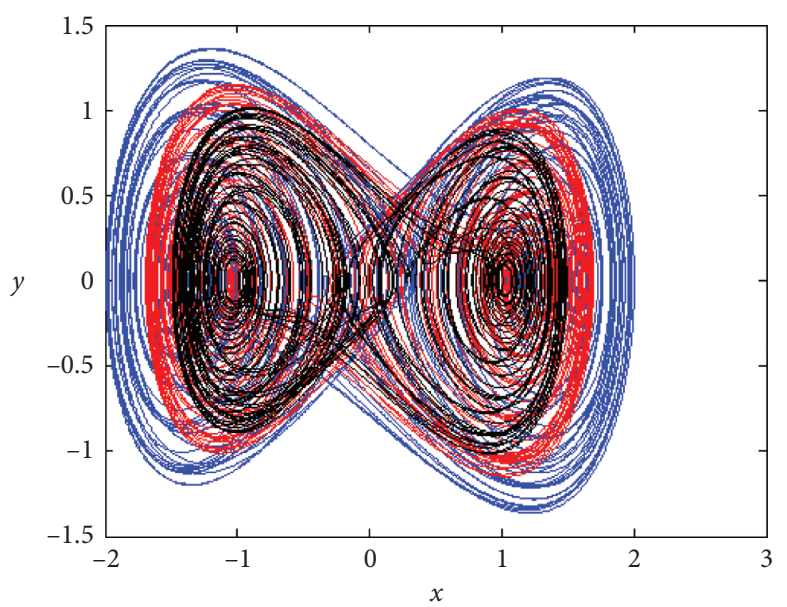

(a)

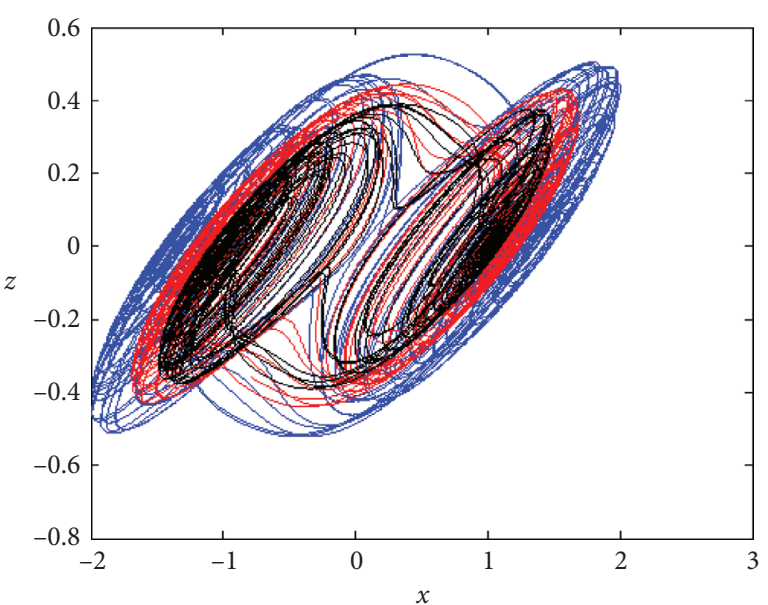

(b)

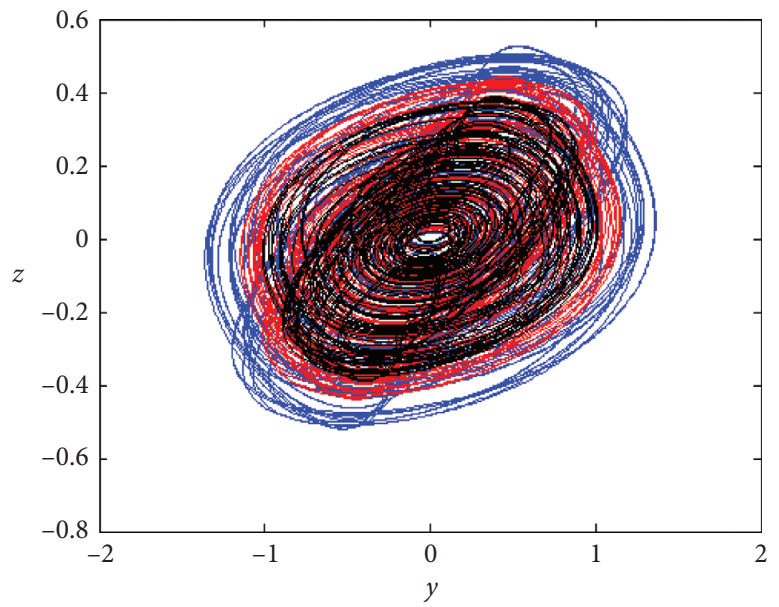

(c)

Figure 20: Representation of the amplitude control of the variables, in the phase space, for three different values of the amplitude control parameter: the amplitudes of the curves in blue, red, and black are obtained for $0.5,0.7$, and 0.9 , respectively. The other parameters $b, w_{f}, k$, and $m$ are set to $0.3,0.5,1.6$, and 0.0 , respectively. 


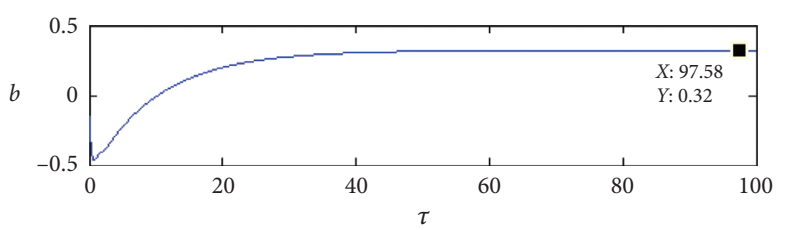

(a)

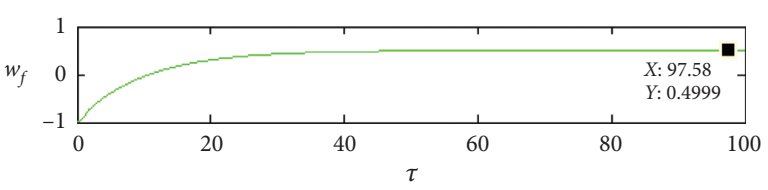

(b)

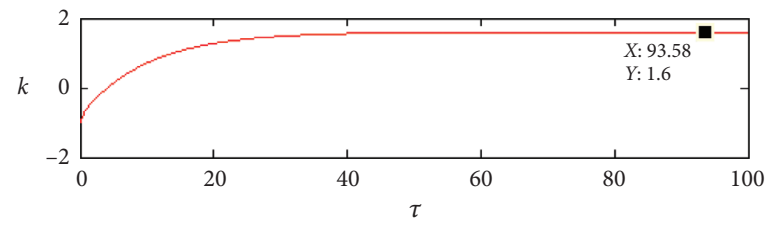

(c)

Figure 21: Temporal evolutions of the estimated gains of $b, w_{f}$, and $k$ converging asymptotically to (a) 0.32 (in blue), (b) 0.5 (in green), and (c) 1.6 (in red), respectively.

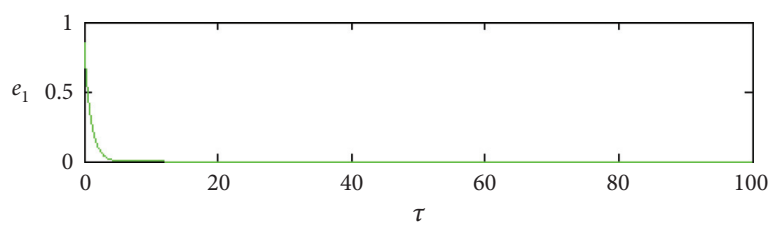

(a)

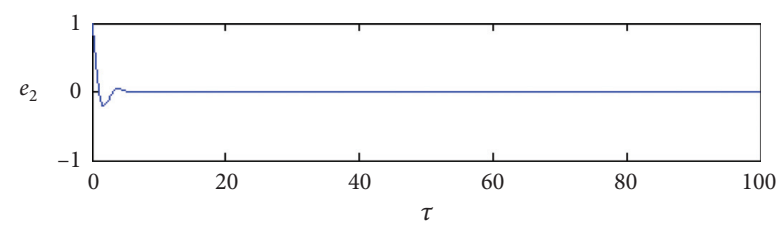

(b)

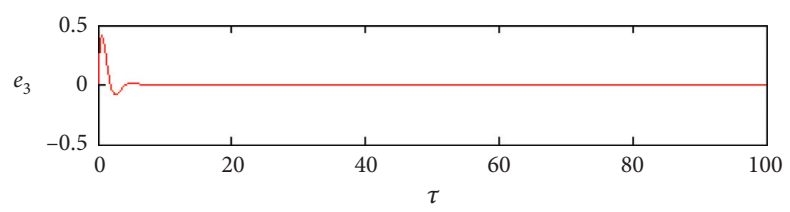

(c)

Figure 22: Temporal evolutions of the errors between the two subsystems (11) and (12) (master and slave), starting from different initial conditions: convergence of the errors (a) $e_{1}$ (in green), (b) $e_{2}$ (in blue), and (c) $e_{3}$ (in red) towards zero.

By differentiating the Lyapunov candidate function (26) along the error trajectory (27), we have

$$
\frac{d V}{\mathrm{~d} \tau}=e_{1} \dot{e}_{1}+e_{2} \dot{e}_{2}+e_{3} \dot{e}_{3}+e_{b} \dot{e}_{b}+e_{w_{f}} \dot{e}_{w_{f}}+e_{k} \dot{e}_{k}
$$

which yields

$$
\begin{aligned}
\dot{V}= & -k_{1} e_{1}^{2}-k_{2} e_{2}^{2}-k_{3} e_{3}^{2}+\left(\dot{e}_{b}+e_{2}^{2}\right) e_{b} \\
& +\left(\dot{e}_{w_{f}}+e_{3}\left(e_{2}-e_{3}\right)\right) e_{w_{f}}+\left(e_{k}-e_{2} e_{3}\right) e_{k} .
\end{aligned}
$$

The subsystems (18) and (19) will be synchronized if (29) is satisfied.

$$
\dot{V}<0 .
$$

The condition for (29) to be satisfied is specified by the following expression:

$$
\left\{\begin{array}{l}
\dot{e}_{b}=-e_{2}^{2}, \\
\dot{e}_{w_{f}}=-e_{3}\left(e_{2}-e_{3}\right), \\
\dot{e}_{k}=e_{2} e_{3} .
\end{array}\right.
$$

Figure 21 shows the evolutions of the estimated parameters $b, w_{f}$, and $k$ converging asymptotically to the values $0.32,0.5$, and 1.6 , respectively. On the other hand, Figure 22 shows evolutions of the errors $e_{1}, e_{2}$, and $e_{3}$ which converge asymptotically towards zero with time. These evolutions prove that the subsystems (18) and (19) are well synchronized. For these numerical simulations, we have considered the initial conditions $\left(x_{1}(0), y_{1}(0), z_{1}(0)\right)=$ $(1,-1,1)$ and $\left(x_{2}(0), y_{2}(0), z_{2}(0)\right)=(1,0,1)$ with the values of the gains $\left(k_{1}, k_{2}, k_{3}\right)=(1,1,0)$. For parameter values, $b=0.32, w_{f}=0.5, m=0$, and $k=1$.6. At the starting point, estimated parameters are $\left(\widehat{b}(0), \widehat{w}_{f}(0), \quad \widehat{k}(0)\right)=$ $(-0.15,-1,-1)$.

\section{Conclusion}

This work focused on the effects of symmetric and asymmetric nonlinearity on the dynamics of a third-order chaotic system, namely, the Duffing-Holmes autonomous oscillator. This has been investigated by supplementing a parametric quadratic term $\left(m x^{2}\right)$ to the cubic nonlinear term $\left(-x^{3}\right)$ of the existing third-order autonomous Duffing-Holmes 
system [42]. An electronic circuit analog to the proposed system has also been designed. Using the Routh-Hurwitz criteria, stability conditions of the fixed points have been established and the existence of a Hopf bifurcation is obtained. The dynamics of the system has been studied by considering two control parameters $(b$ and $k$ ) for discrete values of the parameter $m$. The parameter $m$ made it possible to adjust the symmetry of the system by modifying nontrivial equilibrium points. With $b$ as control parameter, the system exhibits a coexistence of symmetric bifurcation diagrams, confirmed by superposition of the Lyapunov exponent diagrams, phase portraits, and others when $m=0$. When $m \neq 0$, symmetry is destroyed. With $k$ as control parameter, dynamics of the system is still symmetric in the case of $m=0$. This has been confirmed by the superposition of bifurcation diagrams obtained from different initial conditions. With this, the coexistence of four and six attractors in a set of symmetric pairs has been revealed at certain points of the bifurcation diagrams. The cross sections of the basins of attraction have been represented in these different points of coexistence. For the case of $m \neq 0$ (with $k$ as control parameter), the system exhibits asymmetry behavior confirmed by bifurcation diagrams obtained from different initial conditions. These symmetric breakdowns (asymmetry) are related to the movements of the nontrivial equilibria, caused by the values of the parameter $m$. The representations of the dynamics of the system according to its parameters and the Lyapunov exponents revealed the presence of periodic and chaotic regions. The results from the analog simulations in PSpice show good agreement with the numerical results. The results obtained under total amplitude control (TAC) and synchronization analyses conducted using the adaptive method prove that this new system is suitable for applications in various fields of engineering such as encryption of images [73], secure communication [74], and random bit generator [75].

The model reported in this work is a typical example of a 3D system with three rest points and a nonlinear function with a region of negative slope. Also, we conjecture that the results obtained in this work may also be found in the Chua circuit, Shinriki oscillator, and jerk system (with cubic, hyperbolic sine, or hyperbolic tangent) just to cite a few, hence the pertinence and relevance of our study.

\section{Data Availability}

No data were used to support this study.

\section{Conflicts of Interest}

The authors declare that there are no conflicts of interest.

\section{Acknowledgments}

Isaac Sami Doubla would like to thank Mr. L. Kamdjeu Kengne (University of Dschang, Cameroon) for the helpful discussions.

\section{References}

[1] S. Dadras, H. R. Momeni, and G. Qi, "Analysis of a new 3D smooth autonomous system with different wing chaotic attractors and transient chaos," Nonlinear Dynamics, vol. 62, no. 1-2, pp. 391-405, 2010.

[2] Z. Njitacke and J. Kengne, "Complex dynamics of a 4D Hopfield neural networks (HNNs) with a nonlinear synaptic weight: coexistence of multiple attractors and remerging Feigenbaum trees," AEU-International Journal of Electronics and Communications, vol. 93, pp. 242-252, 2018.

[3] S. Doubla Isaac, Z. T. Njitacke, and J. Kengne, "Effects of low and high neuron activation gradients on the dynamics of a simple 3D hopfield neural network," International Journal of Bifurcation and Chaos, vol. 30, no. 11, Article ID 2050159, 2020.

[4] Z. T. Njitacke, I. S. Doubla, S. Mabekou, and J. J. C. Kengne, "Hidden electrical activity of two neurons connected with an asymmetric electric coupling subject to electromagnetic induction: coexistence of patterns and its analog implementation," Solitons, and Fractals, vol. 137, Article ID 109785, 2020.

[5] Z. T. Njitacke, S. D. Isaac, J. Kengne, A. N. Negou, and G. D. J. T. E. P. J. S. T. Leutcho, "Extremely rich dynamics from hyperchaotic Hopfield neural network: hysteretic dynamics, parallel bifurcation branches, coexistence of multiple stable states and its analog circuit implementation," vol. 229, pp. 1133-1154, 2020.

[6] Z. Tabekoueng Njitacke, I. Sami Doubla, J. Kengne, and A. J. C. A. I. J. o.N. S. Cheukem, "Coexistence of firing patterns and its control in two neurons coupled through an asymmetric electrical synapse," vol. 30, no. 2, Article ID 023101, 2020.

[7] B. Dennis, R. A. Desharnais, J. M. Cushing, and R. F. Costantino, "Nonlinear demographic dynamics: mathematical models, statistical methods, and biological experiments," Ecological Monographs, vol. 65, no. 3, pp. 261-282, 1995.

[8] Z.-H. Guan, F. Huang, and W. Guan, "Chaos-based image encryption algorithm," Physics Letters A, vol. 346, no. 1-3, pp. 153-157, 2005.

[9] R. M. Nguimdo, G. Verschaffelt, J. Danckaert, X. Leijtens, J. Bolk, and G. Van der Sande, "Fast random bits generation based on a single chaotic semiconductor ring laser," Optics Express, vol. 20, no. 27, pp. 28603-28613, 2013.

[10] S. Wang, J. Kuang, J. Li, Y. Luo, H. Lu, and G. Hu, "Chaosbased secure communications in a large community," Physical Review E, vol. 66, no. 6, Article ID 065202, 2002.

[11] J. Kengne, "Coexistence of chaos with hyperchaos, period-3 doubling bifurcation, and transient chaos in the hyperchaotic oscillator with gyrators," International Journal of Bifurcation and Chaos, vol. 25, no. 4, Article ID 1550052, 2015.

[12] A. S. de Paula, M. A. Savi, and F. H. I. Pereira-Pinto, "Chaos and transient chaos in an experimental nonlinear pendulum," Journal of Sound and Vibration, vol. 294, no. 3, pp. 585-595, 2006.

[13] Z. Njitacke and L. K. Kengne, "Antimonotonicity, chaos and multiple coexisting attractors in a simple hybrid diode-based jerk circuit," Chaos, Solitons \& Fractals, vol. 105, pp. 77-91, 2017.

[14] J. Kengne, A. N. Negou, and D. Tchiotsop, "Antimonotonicity, chaos and multiple attractors in a novel autonomous memristor-based jerk circuit," Nonlinear Dynamics, vol. 88, no. 4, pp. 2589-2608, 2017. 
[15] J. Kengne, V. F. Signing, J. Chedjou, and G. Leutcho, "Nonlinear behavior of a novel chaotic jerk system: antimonotonicity, crises, and multiple coexisting attractors," International Journal of Dynamics and Control, vol. 6, no. 2, pp. 468-485, 2017.

[16] Z. Njitacke, T. Fozin, L. K. Kengne, G. Leutcho, E. M. Kengne, and J. Kengne, "Multistability and its annihilation in the Chua's oscillator with piecewise-linear nonlinearity," Chaos Theory and Applications, vol. 2, no. 2, pp. 77-89, 2020.

[17] S. Jafari, J. Sprott, and F. Nazarimehr, "Recent new examples of hidden attractors," The European Physical Journal Special Topics, vol. 224, no. 8, pp. 1469-1476, 2015.

[18] V. F. Signing and J. Kengne, "Reversal of period-doubling and extreme multistability in a novel $4 \mathrm{D}$ chaotic system with hyperbolic cosine nonlinearity," International Journal of Dynamics and Control, vol. 7, no. 2, pp. 439-451, 2018.

[19] A. N. Mouelas, T. F. Fozin, R. Kengne, J. Kengne, H. Fotsin, and B. Essimbi, "Extremely rich dynamical behaviors in a simple nonautonomous Jerk system with generalized nonlinearity: hyperchaos, intermittency, offset-boosting and multistability," International Journal of Dynamics and Control, vol. 8, no. 1, pp. 51-69, 2019.

[20] M. E. Cimen, Z. B. Garip, M. A. Pala, A. F. Boz, and A. Akgul, "Modelling of a chaotic system motion in video with artilficial neural networks," Chaos Theory and Applications, vol. 1, no. 1, pp. $38-50,2018$.

[21] A. S. K. Tsafack, R. Kengne, A. Cheukem, J. R. M. Pone, and G. Kenne, "Chaos control using self-feedback delay controller and electronic implementation in IFOC of 3-phase induction motor," Chaos Theory and Applications, vol. 2, no. 1, pp. 40-48, 2020.

[22] Q. Lai, T. Nestor, J. Kengne, and X.-W. Zhao, "Coexisting attractors and circuit implementation of a new 4D chaotic system with two equilibria," Chaos, Solitons \& Fractals, vol. 107, pp. 92-102, 2018.

[23] C. Volos, A. Akgul, V.-T. Pham, I. Stouboulos, and I. Kyprianidis, "A simple chaotic circuit with a hyperbolic sine function and its use in a sound encryption scheme," Nonlinear Dynamics, vol. 89, no. 2, pp. 1047-1061, 2017.

[24] Q. Lai and S. Chen, "Coexisting attractors generated from a new 4D smooth chaotic system," International Journal of Control, Automation and Systems, vol. 14, no. 4, pp. 11241131, 2016.

[25] R. K. Upadhyay, "Multiple attractors and crisis route to chaos in a model food-chain," Chaos, Solitons \& Fractals, vol. 16, no. 5, pp. 737-747, 2003.

[26] J. M. Cushing, S. M. Henson, and C. C. Blackburn, "Multiple mixed-type attractors in a competition model," Journal of Biological Dynamics, vol. 1, no. 4, pp. 347-362, 2007.

[27] C. Masoller, "Coexistence of attractors in a laser diode with optical feedback from a large external cavity," Physical Review A, vol. 50, no. 3, p. 2569, 1994.

[28] Q. Lai, B. Norouzi, and F. Liu, "Dynamic analysis, circuit realization, control design and image encryption application of an extended Lü system with coexisting attractors," Chaos, Solitons \& Fractals, vol. 114, pp. 230-245, 2018.

[29] Q. Lai, P. D. K. Kuate, F. Liu, and H. H.-C. Iu, "An extremely simple chaotic system with infinitely many coexisting attractors," IEEE Transactions on Circuits and Systems II: Express Briefs, vol. 67, no. 6, pp. 1129-1133, 2019.

[30] Q. Lai, Z. Wan, P. D. K. Kuate, and H. Fotsin, "Coexisting attractors, circuit implementation and synchronization control of a new chaotic system evolved from the simplest memristor chaotic circuit," Communications in Nonlinear
Science and Numerical Simulation, vol. 89, Article ID 105341, 2020.

[31] Q. Lai, Z. Wan, and P. D. K. Kuate, "Modelling and circuit realisation of a new no-equilibrium chaotic system with hidden attractor and coexisting attractors," Electronics Letters, vol. 56, no. 20, 2020.

[32] Y. Adiyaman, S. Emiroglu, M. K. Uçar, and M. Yildiz, "Dynamical analysis, electronic circuit design and control application of a different chaotic system," Chaos Theory and Applications, vol. 2, no. 1, pp. 8-14, 2020.

[33] K. G. Honoré, N. N. L. Parfait, C. Ainamon, and S. T. Kingni, "Theoretical and experimental investigations of a jerk circuit with two parallel diodes," Chaos Theory and Applications, vol. 2, no. 2, pp. 52-57, 2020.

[34] A. N. Negou, J. J. A.-I. J. o.E. Kengne, and Communications, "Dynamic analysis of a unique jerk system with a smoothly adjustable symmetry and nonlinearity: reversals of period doubling," Offset Boosting and Coexisting Bifurcations, vol. 90, pp. 1-19, 2018.

[35] J. Kengne, Z. Njitacke, and H. Fotsin, "Dynamical analysis of a simple autonomous jerk system with multiple attractors," Nonlinear Dynamics, vol. 83, no. 1-2, pp. 751-765, 2016.

[36] J. Kengne, S. Njikam, and V. F. Signing, "A plethora of coexisting strange attractors in a simple jerk system with hyperbolic tangent nonlinearity," Chaos, Solitons \& Fractals, vol. 106, pp. 201-213, 2018.

[37] J. Kengne and R. L. T. Mogue, "Dynamic analysis of a novel jerk system with composite tanh-cubic nonlinearity: chaos, multi-scroll, and multiple coexisting attractors," International Journal of Dynamics and Control, vol. 7, no. 1, pp. 112-133, 2020.

[38] G. Leutcho, J. Kengne, and L. K. Kengne, "Dynamical analysis of a novel autonomous 4-D hyperjerk circuit with hyperbolic sine nonlinearity: chaos, antimonotonicity and a plethora of coexisting attractors," Chaos, Solitons \& Fractals, vol. 107, pp. 67-87, 2018.

[39] R. M. Tagne, J. Kengne, and A. N. Negou, "Multistability and chaotic dynamics of a simple Jerk system with a smoothly tuneable symmetry and nonlinearity," International Journal of Dynamics and Control, vol. 7, no. 2, pp. 476-495, 2018.

[40] G. D. Leutcho and J. Kengne, "A unique chaotic snap system with a smoothly adjustable symmetry and nonlinearity: chaos, offset-boosting, antimonotonicity, and coexisting multiple attractors," Chaos, Solitons \& Fractals, vol. 113, pp. 275-293, 2018.

[41] J. Kengne, R. L. T. Mogue, T. F. Fozin, and A. N. K. Telem, "Effects of symmetric and asymmetric nonlinearity on the dynamics of a novel chaotic jerk circuit: coexisting multiple attractors, period doubling reversals, crisis, and offset boosting," Chaos, Solitons \& Fractals, vol. 121, pp. 63-84, 2019.

[42] E. Lindberg, E. Tamaseviciute, G. Mykolaitis et al., "Autonomous third-order duffing-holmes type chaotic oscillator," in Proccedings of the 2009 European Conference on Circuit Theory and Design, pp. 663-666, IEEE, Antalya, Turkey, Augus 2009.

[43] E. Ott, Chaos in Dynamical Systems, Cambridge University Press, Cambridge, UK, 2002.

[44] R. C. Hilborn, Chaos and Nonlinear Dynamics: An Introduction for Scientists and Engineers, Oxford University Press on Demand, Oxford, UK, 2000.

[45] H. Dang-Vu and C. Delcarte, Bifurcations et Chaos: Une Introduction à la Dynamique Contemporaine Avec des 
Programmes en Pascal, Fortran et Mathematica, Ellipses Ed. Marketing, 2000.

[46] W. Zhou, Y. Xu, H. Lu, and L. Pan, "On dynamics analysis of a new chaotic attractor," Physics Letters A, vol. 372, no. 36, pp. 5773-5777, 2002.

[47] S. Dadras and H. R. Momeni, "A novel three-dimensional autonomous chaotic system generating two, three and fourscroll attractors," Physics Letters A, vol. 373, no. 40, pp. 3637-3642, 2009.

[48] Z. Wei and Q. Yang, "Dynamical analysis of a new autonomous 3-D chaotic system only with stable equilibria," Nonlinear Analysis: Real World Applications, vol. 12, no. 1, pp. 106-118, 2011.

[49] F. Yu and C. Wang, "A novel three dimension autonomous chaotic system with a quadratic exponential nonlinear term," Engineering, Technology \& Applied Science Research, vol. 2, no. 2, pp. 209-215, 2020.

[50] S. Strogatz, Nonlinear Dynamics and Chaos, Addison Wesley, Reading, MA, USA, 1994.

[51] A. Wolf, J. B. Swift, H. L. Swinney, and J. A. Vastano, "Determining Lyapunov exponents from a time series," Physica D: Nonlinear Phenomena, vol. 16, no. 3, pp. 285-317, 1985.

[52] J. H. Argyris, G. Faust, M. Haase, and R. Friedrich, An Exploration of Dynamical Systems and Chaos: Completely Revised and Enlarged, Springer, Berlin, Germany, 2nd edition, 2015.

[53] N. H. Alombah, H. Fotsin, and K. Romanic, "Coexistence of multiple attractors, metastable chaos and bursting oscillations in a multiscroll memristive chaotic circuit," International Journal of Bifurcation and Chaos, vol. 27, no. 5, Article ID 1750067, 2017.

[54] S. Yu, W. K. Tang, J. Lu, and G. Chen, "Generation of $n \times m$ Wing Lorenz-like attractors from a modified Shimizu-Morioka model," IEEE Transactions on Circuits and Systems II: Express Briefs, vol. 55, no. 11, pp. 1168-1172, 2008.

[55] W. Hao-Xiang, C. Guo-Liang, M. Sheng, and T. Li-Xin, "Nonlinear feedback control of a novel hyperchaotic system and its circuit implementation," Chinese Physics B, vol. 19, no. 3, Article ID 030509.

[56] C. Li and J. Sprott, "Amplitude control approach for chaotic signals,” Nonlinear Dynamics, vol. 73, no. 3, pp. 1335-1341, 2013.

[57] C. Li, J. C. Sprott, W. Thio, and H. Zhu, "A new piecewise linear hyperchaotic circuit," IEEE Transactions on Circuits and Systems II: Express Briefs, vol. 61, no. 12, pp. 977-981, 2014.

[58] C. Li, J. C. Sprott, and W. Thio, "Linearization of the lorenz system," Physics Letters A, vol. 379, no. 10-11, pp. 888-893, 2015.

[59] C. Li and J. Sprott, "Finding coexisting attractors using amplitude control," Nonlinear Dynamics, vol. 78, no. 3, pp. 2059-2064, 2014.

[60] C. Li, J. C. Sprott, Z. Yuan, and H. Li, "Constructing chaotic systems with total amplitude control," International Journal of Bifurcation and Chaos, vol. 25, no. 10, Article ID 1530025, 2015.

[61] A. Akgul, C. Li, and I. Pehlivan, "Amplitude control analysis of a four-wing chaotic attractor, its electronic circuit designs and microcontroller-based random number generator," Journal of Circuits, Systems and Computers, vol. 26, no. 12, Article ID 1750190, 2017.

[62] A. A. Zaher and A. Abu-Rezq, "On the design of chaos-based secure communication systems," Communications in
Nonlinear Science and Numerical Simulation, vol. 16, no. 9, pp. 3721-3737, 2011.

[63] J. L. Mata-Machuca, R. Martínez-Guerra, R. Aguilar-López, and C. Aguilar-Ibañez, "A chaotic system in synchronization and secure communications," Communications in Nonlinear Science and Numerical Simulation, vol. 17, no. 4, pp. 17061713, 2019.

[64] B. Nana, P. Woafo, and S. Domngang, "Chaotic synchronization with experimental application to secure communications," Communications in Nonlinear Science and Numerical Simulation, vol. 14, no. 5, pp. 2266-2276, 2009.

[65] N. Smaoui, A. Karouma, and M. Zribi, "Secure communications based on the synchronization of the hyperchaotic Chen and the unified chaotic systems," Communications in Nonlinear Science and Numerical Simulation, vol. 16, no. 8, pp. 3279-3293, 2011.

[66] J. Grzybowski, M. Rafikov, and J. M. Balthazar, "Synchronization of the unified chaotic system and application in secure communication," Communications in Nonlinear Science and Numerical Simulation, vol. 14, no. 6, pp. 2793-2806, 2009.

[67] B. Nana and P. Woafo, "Synchronized states in a ring of four mutually coupled oscillators and experimental application to secure communications," Communications in Nonlinear Science and Numerical Simulation, vol. 16, no. 4, pp. 17251733, 2011.

[68] A. C. Luo, "A theory for synchronization of dynamical systems," Communications in Nonlinear Science and Numerical Simulation, vol. 14, no. 5, pp. 1901-1951, 2009.

[69] X.-Y. Wang and B. Fan, "Generalized projective synchronization of a class of hyperchaotic systems based on state observer," Communications in Nonlinear Science and Numerical Simulation, vol. 17, no. 2, pp. 953-963, 2012.

[70] L. Xiao-Wen and Z. Zhi-Gang, "Phase synchronization of coupled Rossler oscillators: amplitude effect," Communications in Theoretical Physics, vol. 47, no. 2, p. 265, 2007.

[71] N. Smaoui, A. Karouma, and M. Zribi, "Synchronization of the hyperchaotic Lüsystems using a sliding mode controller," Kuwait Journal of Science, vol. 38, no. 2A, pp. 69-91, 2011.

[72] J. H. Park, "Adaptive synchronization of hyperchaotic Chen system with uncertain parameters," Chaos, Solitons \& Fractals, vol. 26, no. 3, pp. 959-964, 2005.

[73] G. Alvarez and S. Li, "Some basic cryptographic requirements for chaos-based cryptosystems," International Journal of Bifurcation and Chaos, vol. 16, no. 8, pp. 2129-2151, 2016.

[74] E. Tlelo-Cuautle, V. Carbajal-Gomez, P. Obeso-Rodelo, J. Rangel-Magdaleno, and J. C. Nuñez-Perez, "FPGA realization of a chaotic communication system applied to image processing," Nonlinear Dynamics, vol. 82, no. 4, pp. 18791892, 2015.

[75] Z. Wang, A. Akgul, V.-T. Pham, and S. Jafari, "Chaos-based application of a novel no-equilibrium chaotic system with coexisting attractors," Nonlinear Dynamics, vol. 89, no. 3, pp. 1877-1887, 2017. 